\title{
41. LATE NEOGENE PALEOCEANOGRAPHIC EVOLUTION OF SITE 590: SOUTHWEST PACIFIC 1
}

\author{
Kristin M. Elmstrom and James P. Kennett, Graduate School of Oceanography, University of Rhode Island ${ }^{2}$
}

\begin{abstract}
High biogenic sedimentation rates in the late Neogene at DSDP Site $590(1293 \mathrm{~m})$ provide an exceptional opportunity to evaluate late Neogene (late Miocene to latest Pliocene) paleoceanography in waters transitional between temperate and warm-subtropical water masses. Oxygen and carbon isotope analyses and quantitative planktonic foraminiferal data have been used to interpret the late Neogene paleoceanographic evolution of this site.

Faunal and isotopic data from Site 590 show a progression of paleoceanographic events between 6.7 and $4.3 \mathrm{Ma}$, during the latest Miocene and early Pliocene. First, a permanent depletion in both planktonic and benthic foraminiferal $\delta^{13} \mathrm{C}$, between 6.7 and $6.2 \mathrm{Ma}$, can be correlated to the globally recognized late Miocene carbon isotope shift. Second, a $0.5 \%$ enrichment in benthic foraminiferal $\delta^{18} \mathrm{O}$ between 5.6 and $4.7 \mathrm{Ma}$ in the latest Miocene to early Pliocene corresponds to the latest Miocene oxygen isotopic enrichment at Site 284, located in temperate waters south of Site 590 . This enrichment in $\delta^{18} \mathrm{O}$ coincides with a time of cool surface waters, as is suggested by high frequencies of Neogloboquadrina pachyderma and low frequencies of the warmer-water planktonic foraminifers, as well as by an enrichment in planktonic foraminiferal $\delta^{18} \mathrm{O}$ relative to the earlier Miocene. By $4.6 \mathrm{Ma}$, benthic foraminiferal $\delta^{18} \mathrm{O}$ values become depleted and remain fairly stable until about $3.8 \mathrm{Ma}$.

The early Pliocene $(\sim 4.3$ to $3.2 \mathrm{Ma})$ is marked by a significant increase in biogenic sedimentation rates $(37.7$ to 83.3 $\mathrm{m} / \mathrm{m}$.y.). During this time, heaviest values in planktonic foraminiferal $\delta^{18} \mathrm{O}$ are associated with a decrease in the gradient between surface and intermediate-water $\delta^{13} \mathrm{C}$ and $\delta^{18} \mathrm{O}$, a $1.0 \%$ depletion in the $\delta^{13} \mathrm{C}$ of two species of planktonic foraminifers, and a mixture of warm and cool planktonic foraminiferal elements. These data suggest that localized upwelling at the Subtropical Divergence produced an increase in surface-water productivity during the early Pliocene.

A two-step enrichment in benthic foraminiferal $\delta^{18} \mathrm{O}$ occurs in the late Pliocene sequence at Site 590. A $0.3 \% 0$ average enrichment at about $3.6 \mathrm{Ma}$ is followed by a $0.5 \%$ enrichment at $2.7 \mathrm{Ma}$. These two events can be correlated with the two-step isotopic enrichment associated with late Pliocene climatic instability and the initiation of Northern Hemisphere glaciation.
\end{abstract}

\section{INTRODUCTION}

Development of the hydraulic piston core (HPC) and the extended core barrel (XCB) have enabled high-quality sedimentary sections to be obtained from the deep sea. Such a high-quality site is DSDP Site 590 , located in the southwest Pacific at $1293 \mathrm{~m}$ on the eastern flank of the Lord Howe Rise (Fig. 1). The site is located under the present-day position of the Subtropical Divergence (Tasman Front; Denham and Crook, 1976; Stanton, 1979, 1981), which separates temperate surface-water masses to the south from warm-subtropical water masses to the north. The front represents the southern extent of the South Pacific subtropical gyre as the East Australian Current turns to the east, passing to the north of New Zealand. Divergence and associated upwelling at the Subtropical Divergence is caused by the interaction of the eastward-flowing gyral circulation with the Lord Howe Rise (Stanton, 1981) and has created high rates of biogenic productivity during the late Neogene (Kennett et al., 1983). The sedimentary sequence cored at Site 590 ranges in age from early Miocene to Recent and is composed of a nannofossil ooze to foraminifer-bearing nannofossil ooze. Three holes were drilled; Hole 590 termi-

\footnotetext{
${ }^{1}$ Kennett, J. P., von der Borch, C. C., et al., Init. Repts. DSDP, 90: Washington (U.S. Govt, Printing Office).

2 Address: Graduate School of Oceanography, University of Rhode Island, Narragansett, RI 02882 .
}

nated after only three cores were taken; Hole 590A, which extends from the earliest Pleistocene to the late Miocene; and Hole 590B, which extends from the late Pleistocene to the early Miocene.

In this chapter we study the paleoceanographic evolution of Hole 590A from the late Miocene ( 7.0 Ma) to the latest Pliocene $(\sim 1.8 \mathrm{Ma})$, using quantitative changes in planktonic foraminifers and planktonic and benthic foraminiferal stable isotopes. The position of the site on the Subtropical Divergence separating temperate from warm-subtropical faunal assemblages, the shallow depth of deposition, and the excellent core recovery and microfossil preservation make this site ideal for studying late Neogene paleoceanographic evolution.

Although this is an analysis of nearly the entire late Neogene we are particularly interested in those intervals of major paleoclimatic change. These include the latest Miocene, a time of widespread cooling of surface waters and global glacial increase (Kennett, 1967; Ciesielski et al., 1982; Vincent et al., 1980; Loutit, 1981; Kennett et al., 1979; Adams et al., 1977; and Vail et al., 1977), and the late Pliocene, which was associated with further cooling and oceanic oxygen isotope change resulting from the onset of Northern Hemisphere glaciation (Shackleton and Opdyke, 1977; Kennett et al., 1979; Backman, 1979; Hodell et al., 1983; Shackleton et al., 1984). Much of the early Pliocene was characterized by relative climatic warmth and stability (Shackleton and Kennett, 1975; Kennett, 1978). 


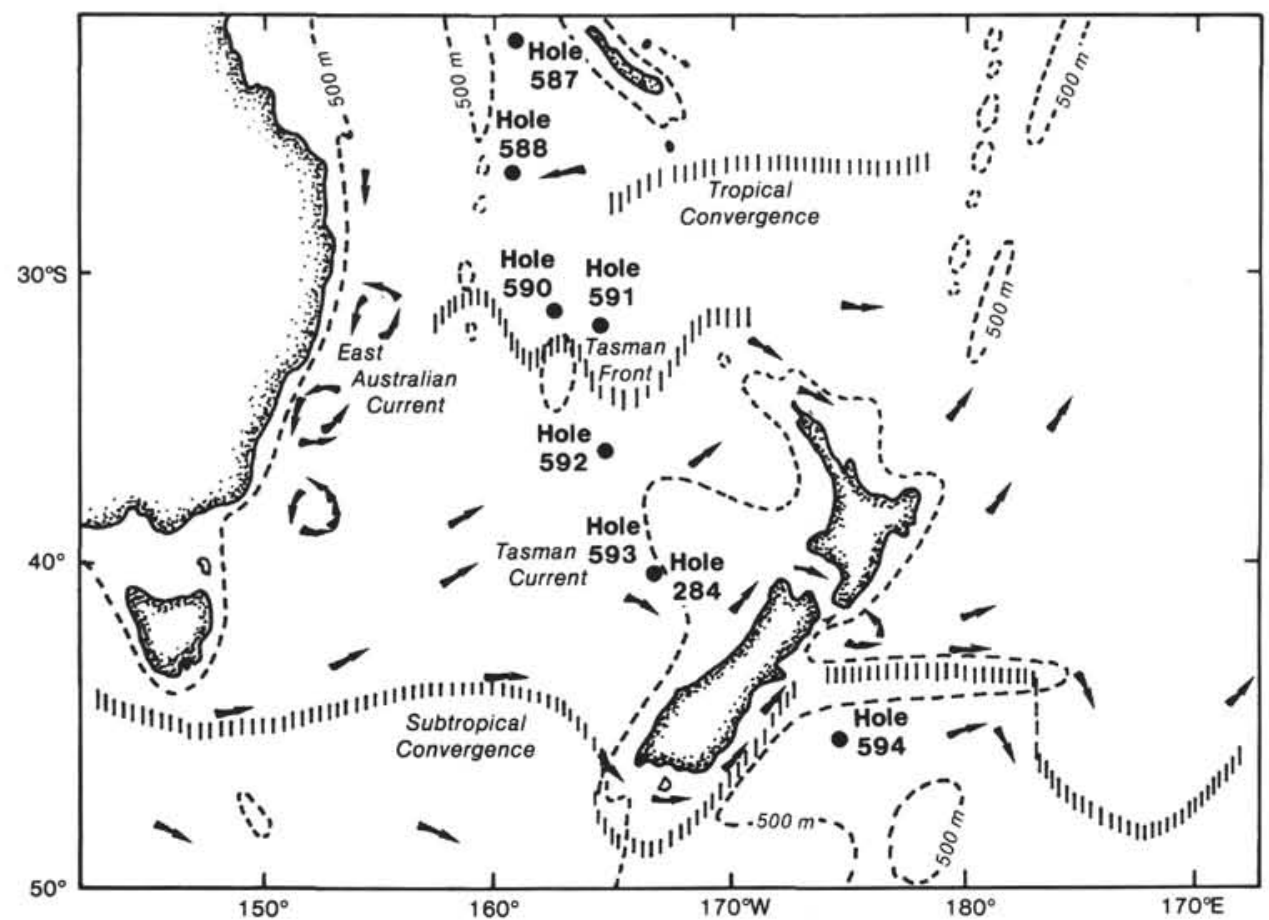

Figure 1. Map showing the location of Site $590(1293 \mathrm{~m})$ and other DSDP sites in the southwest Pacific. Site 590 is located on the eastern flank of the Lord Howe Rise. Shown also are the present-day positions of the Subtropical Divergence (Tasman Front) and the Subtropical Convergence. Isobath (dotted line) is the $1000 \mathrm{~m}$ contour.

\section{BIOSTRATIGRAPHY}

We have used, with some minor variations, the planktonic foraminiferal zones established for Site 590 by the shipboard scientific party (Site 590 site chapter, this volume), which are in turn based upon the zonal scheme of Kennett (1973) (Fig. 2).

The Globorotalia conomiozea Zone is marked by the first abundant appearance of $G$. conomiozea at $225 \mathrm{~m}$, rather than the $240 \mathrm{~m}$ determined in the Site 590 site chapter (this volume). The base of the $G$. puncticulata Zone, marking the Miocene/Pliocene boundary, is based upon the first evolutionary appearance of $G$. puncticulata puncticulata from $G$. puncticulata sphericomiozea at $190 \mathrm{~m}$, as compared to the $194 \mathrm{~m}$ determined in the site chapter (this volume). The first appearance of $G$. pliozea also coincides with the first appearance of $G$. puncticulata and the Miocene/Pliocene boundary, whereas the first appearance of $G$. margaritae occurs $4 \mathrm{~m}$ below the boundary. The base of the $G$. crassaformis Zone is marked by the first appearance of $G$. crassaformis at $165 \mathrm{~m}$. A gap in core recovery occurs between 113 and $101 \mathrm{~m}$, with the base of the $G$. inflata Zone marked by the first appearance of $G$. inflata at $101 \mathrm{~m}$. The base of the $G$. tosaensis Zone is based upon the first appearance of $G$. tosaensis at $80 \mathrm{~m}$. The first appearance of $G$. truncatulinoides in association with $G$. tosaensis marks the base of the $G$. tosaensis-G. truncatulinoides Overlap Zone. The first appearance of $G$. truncatulinoides has often been taken to represent the Pliocene/Pleistocene boundary, although Rio et al. (1984) demonstrate its first appearance in the late Pliocene. However, at Site 590 the first appearance of this species is within nannofossil Zone NN17 of late Pliocene age, and within the early Matuyama Paleomagnetic Chron. The base of the $G$. truncatulinoides-G. tosaensis Overlap Zone is therefore of late Pliocene age.

\section{CHRONOLOGY}

The late Neogene sequence at Site 590 is dated in its upper $81 \mathrm{~m}$ using magnetostratigraphy (Barton and Bloemendal, this volume). A magnetostratigraphy could not be determined for the sequence deeper than $81 \mathrm{~m}$ at Site 590 because of very low intensities of magnetization. Therefore, the chronology for the earlier part of the late Neogene sequence is based upon ages assigned to specific datum levels. In all, a total of 12 datum levels have been used to provide the chronological framework, and to determine sedimentation rates (Fig. 3, 4). Many of these are summarized in the site chapter, (this volume) and are based upon the compilation of Berggren et al. (in press).

The datum levels used to establish our chronology from oldest to youngest are as follows:

1. The globally recognized late Miocene carbon isotope shift (Haq et al., 1980), dated by Loutit and Kennett (1979) using magnetostratigraphy on an uplifted marine sequence in New Zealand, at 6.3 Ma.

2. The first abundant appearance of Globorotalia conomiozea at $225 \mathrm{~m}$, which has been dated at $6.1 \mathrm{Ma}$ by Loutit and Kennett (1979).

3. The base of NN12 at $196 \mathrm{~m}$, which has been assigned an age of $5.2 \mathrm{Ma}$ in the site chapter (this volume). 


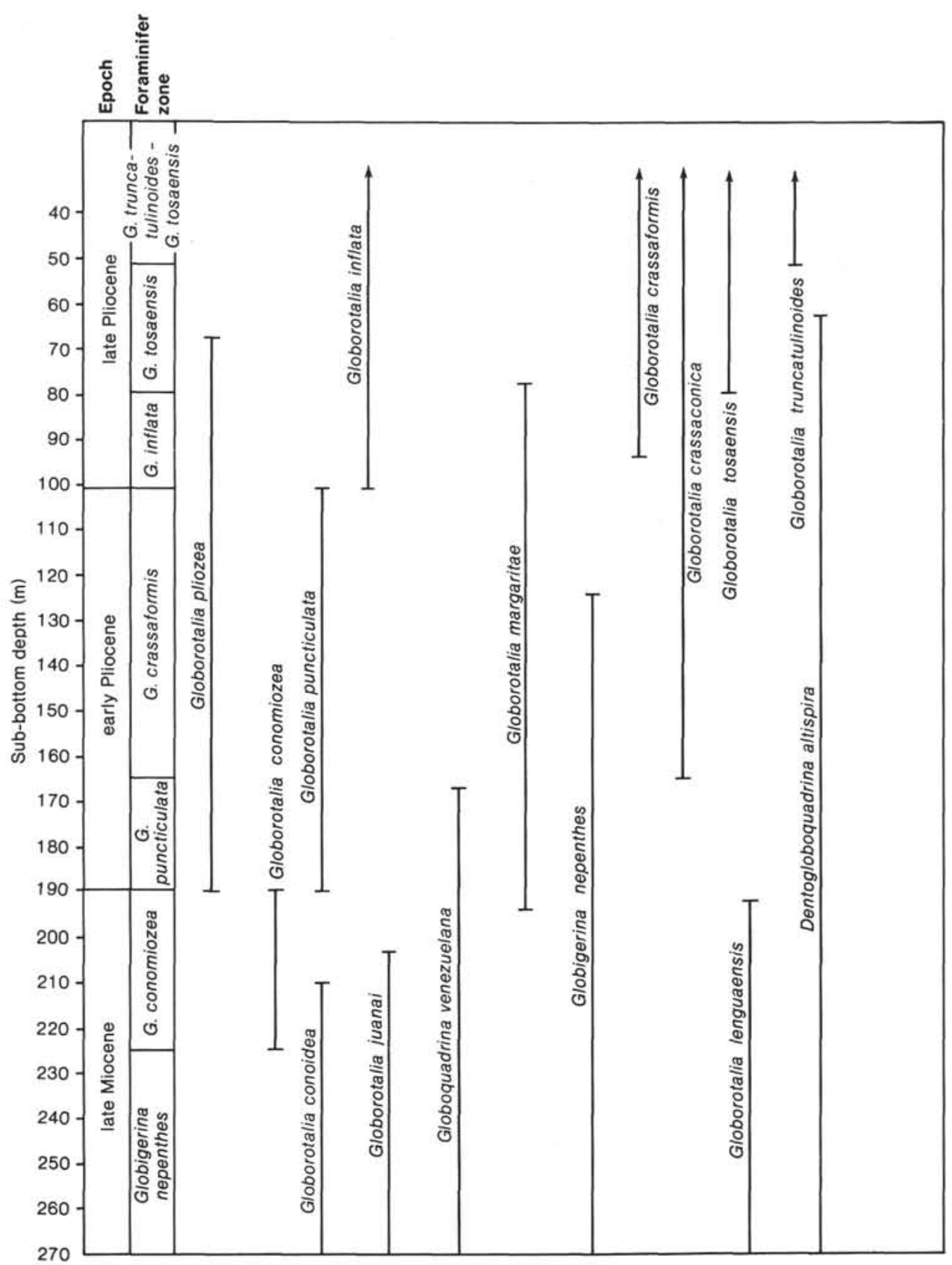

Figure 2. Ranges of certain late Neogene planktonic foraminifers at Site $\mathbf{5 9 0 .}$

4. The base of NN13 at $172 \mathrm{~m}$, which has been assigned an age of $4.6 \mathrm{Ma}$ in the site chapter (this volume).

5. The base of NN14 at $151 \mathrm{~m}$, which has been assigned an age of $4.1 \mathrm{Ma}$ in the site chapter (this volume).

6. The base of NN15 at $131 \mathrm{~m}$, which has been assigned an age of $3.65 \mathrm{Ma}$ in the site chapter (this volume).

7. The Gauss/Gilbert Paleomagnetic Chron boundary at $81.2 \mathrm{~m}$, which has been assigned an age of $3.4 \mathrm{Ma}$ by Barton and Bloemendal (this volume).

8. The base of the Mammoth Paleomagnetic Subchron at $70 \mathrm{~m}$, which has been assigned an age of 3.18 Ma by Barton and Bloemendal (this volume).
9. The top of the Kaena Paleomagnetic Subchron at $62.5 \mathrm{~m}$, which has been assigned an age of $2.92 \mathrm{Ma}$ by Barton and Bloemendal (this volume).

10. The Gauss/Matuyama Paleomagnetic Chron boundary at $47.9 \mathrm{~m}$, which has been assigned an age of $2.47 \mathrm{Ma}$ by Barton and Bloemendal (this volume).

11. The base of the Olduvai Paleomagnetic Subchron at $35.75 \mathrm{~m}$, which has been assigned as age of $1.88 \mathrm{Ma}$ by Barton and Bloemendal (this volume).

12. The top of Olduvai Paleomagnetic Subchron at $33 \mathrm{~m}$, which has been assigned an age of $1.66 \mathrm{Ma}$ by Barton and Bloemendal (this volume).

The use of this chronology shows that sedimentation rates at Site 590 range from 22.5 to $83.3 \mathrm{~m} / \mathrm{m}$.y. (Fig. 4). The high rates $(72.4 \mathrm{~m} / \mathrm{m}$.y.) in early late Miocene may 


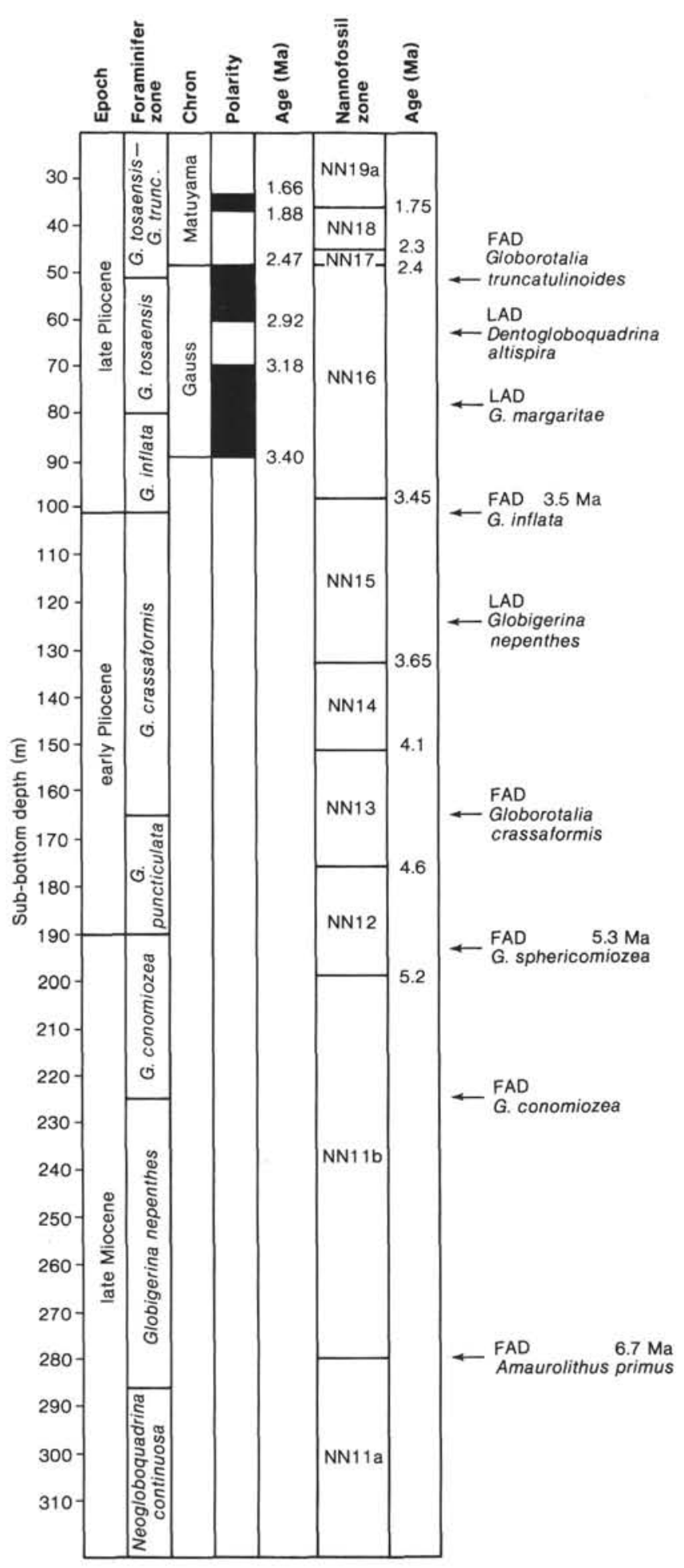

Figure 3. Correlation between planktonic foraminiferal biostratigraphy, calcareous nannofossil biostratigraphy, microfossil datums, and paleomagnetic stratigraphy of Site 590 plotted against depth.

be unreliable because chronological control was poor. The sedimentation rates are based upon the slope of a bestfit regression line ( $>93 \%$ confidence level) through the given datum levels. The curve indicates relatively low sedimentation rates of $37.7 \mathrm{~m} / \mathrm{m}$.y. from the latest Miocene $(\sim 5.6 \mathrm{Ma})$ to the early Pliocene $(\sim 4.2 \mathrm{Ma})$. This is followed by unusually high middle to late Pliocene sedimentation rates of $83.3 \mathrm{~m} / \mathrm{m}$.y. between 4.2 and 3.2 $\mathrm{Ma}$, which the shipboard party (site chapter, this volume) considered to have resulted from very high rates of calcareous nannofossil productivity caused by intensified upwelling at the Subtropical Divergence. At $\sim 3.2$ $\mathrm{Ma}$, sedimentation rates again decrease to lower rates of $22.5 \mathrm{~m} / \mathrm{m} . \mathrm{y}$.

\section{METHODS}

Planktonic foraminiferal counts were made on 112 samples from the latest Miocene to the latest Pliocene. Samples were oven-dried at $60^{\circ} \mathrm{C}$, weighed, disaggregated in a hot Calgon solution, washed over a $63-\mu \mathrm{m}$ Tyler sieve, dried, then reweighed. The $>150-\mu \mathrm{m}$ size fraction was separated and repeatedly placed through a microsplitter to obtain an aliquot of $\sim 300$ specimens that were counted. Thirty-eight species of planktonic foraminifers were identified using the taxonomy of Kennett and Srinivasan (1983). Species frequencies were calculated within the total population.

Oxygen and carbon isotope analyses were performed on both planktonic and benthic foraminifers throughout Hole 590A. Planktonic analyses were performed on Globigerinoides sacculifer in the fraction from 295 to $417-\mu \mathrm{m}$. Material was analyzed from a relatively small size range to decrease potential variability in isotopic composition between different sizes of planktonic foraminifers (Killingley et al., 1981). Analyses were also performed on 15 samples of Globorotalia puncticulata from 154 to $180 \mathrm{~m}$ to provide a comparison between an inferred deeperdwelling form G. puncticulata, and a known shallow-water species, Globigerinoides sacculifer.

Benthic foraminiferal isotopic analyses were performed on Cibicidoides kullenbergi from the $>250-\mu \mathrm{m}$ size fraction. When an adequate number of specimens were available for analyses, very large specimens $(>400-\mu \mathrm{m})$ were not included in the isotopic analyses.

Specimens for isotopic analyses were ultrasonically cleaned in reagent-grade methanol and roasted in vacuo at $400^{\circ} \mathrm{C}$ for $1 \mathrm{hr}$. The samples were then reacted in $100 \%$ orthophosphoric acid at $50^{\circ} \mathrm{C}$. The gas was then subject to three distillations in order to purify the remaining $\mathrm{CO}_{2}$ gas, which was then analyzed for ${ }^{18} \mathrm{O}$ and ${ }^{13} \mathrm{C}$ using an on-line VG Micromass 602D mass spectrometer with an analytical precision of $\pm 0.1 \%$ for both $\delta^{18} \mathrm{O}$ and $\delta^{13} \mathrm{C}$, based upon the analyses of laboratory standard of powdered B-1, which is run at the beginning and end of each day. All isotopic results are expressed as per mil differences from PDB.

\section{FAUNAL RESULTS}

Neogloboquadrina pachyderma is an important component in the planktonic foraminiferal assemblage at Site 590, with frequencies ranging up to $50 \%$ (Fig. 5, 6; Table 1). Highest frequencies of this species occur during the latest Miocene through early Pliocene (6.1 to 4.5 Ma), moderately high frequencies occur in the early Pliocene between 4.0 and $3.3 \mathrm{Ma}$, and lowest frequencies $(<20 \%)$ are generally exhibited during the earlier late Miocene (6.3-6.1 Ma) and again in the latest Pliocene (3.3 to $2.1 \mathrm{Ma}$ ). During most of the late Miocene and early Pliocene, the coiling direction of $N$. pachyderma is dominantly sinistral. One brief interval of dominantly dextral coiling occurs during the late Miocene, centered at about 6.0 Ma. There is a rapid switch from dominantly sinistral to dominantly dextral coiling in the early Pliocene $(\sim 4.3 \mathrm{Ma})$, after which populations of $N$. pachyderma are always dominantly dextrally coiled.

Frequency oscillations are also shown in Figure 6 for a group of species that are considered to have a warmerwater tolerance: Globigerinoides obliquus, G. sacculifer, G. ruber, Globorotalia menardii, Neogloboquadrina acostaensis, N. humerosa, Dentogloboquadrina altispira, and 


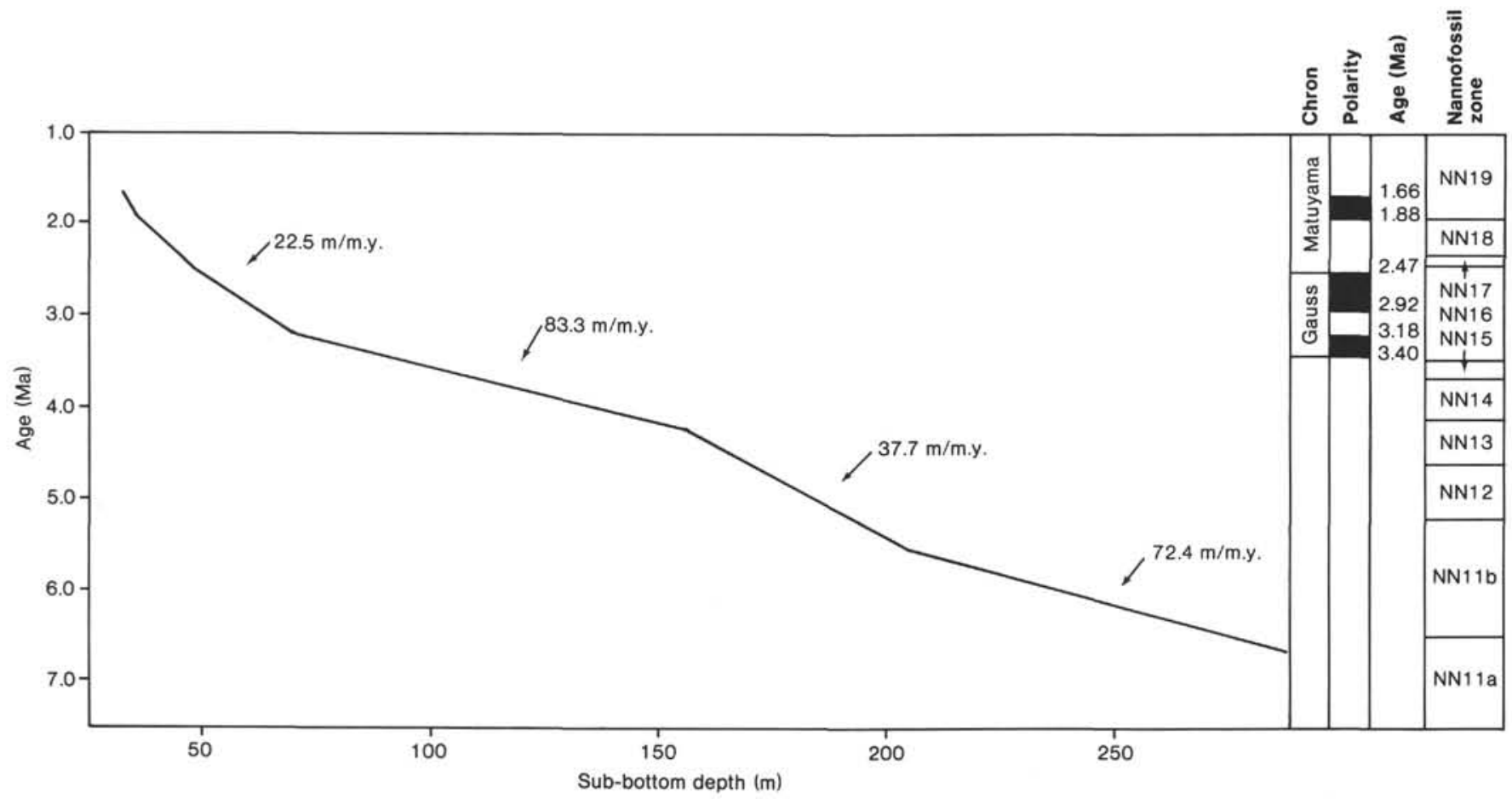

Figure 4. Sedimentation rate diagram for Site 590 based upon 12 datum levels.

Globorotalia margaritae (Kennett and Srinivasan, 1983). Warm-subtropical faunal elements exhibit particularly low frequencies in the latest Miocene through the early Pliocene (5.6 to $4.3 \mathrm{Ma})$, and in the latest Pliocene ( 3.3 to $2.1 \mathrm{Ma}$ ). Particularly high frequencies of the warm-subtropical fauna occur through the remaining parts of the early Pliocene $(4.3$ to $3.3 \mathrm{Ma}$ ) and the earlier part of the late Miocene (6.3 to $5.6 \mathrm{Ma})$. The switch in the coiling direction of $N$. pachyderma from dominantly sinistral to dominantly dextral occurs in the early Pliocene coincident with the increase in frequencies of the warm-subtropical fauna and decrease in frequencies of the coldwater form, $N$. pachyderma.

Three important components of the assemblage (Globigerina woodi, $G$. bulloides, and $G$. falconensis) exhibit no clear faunal changes throughout the sequence. Paleoceanographic changes at this latitude were not large enough to cause important frequency oscillations in these forms (Fig. 7) and hence they are of no value as paleoceanographic monitors in this site.

The Globorotalia (Globoconella) lineage (Kennett and Srinivasan, 1983) is another important element within the assemblage at certain times in the late Neogene (Fig. 7). The lineage consists of an evolutionary transition from $G$. conoidea to $G$. conomiozea to $G$. puncticulata sphericomiozea to $G$. puncticulata puncticulata to $G$. inflata. The evolution is centered in temperate waters, and each species is assumed to have had similar environmental preferences during the late Neogene. Very high frequencies in the early Miocene (6.3 to 6.1 Ma) are followed by relatively low values during the latest Miocene and early Pliocene (6.1 to $3.9 \mathrm{Ma}$ ). Two step-like increases occur in the Globoconella lineage, one in the late early Pliocene ( 3.9 Ma) and the other in the late
Pliocene ( 3.4 Ma) when the lineage reaches maximum frequencies in the form of $G$. inflata.

Globigerinita glutinata exhibits an increase in frequency across the Miocene/Pliocene boundary and again in the late Pliocene. Globorotalia margaritae and Orbulina universa both rapidly disappear for a brief interval during the early Pliocene ( 4.0 to $4.5 \mathrm{Ma}$ ), whereas Globorotalia scitula, a species known to live deeper in the water column (Williams et al., 1977), increases in frequencies during the same interval.

\section{OXYGEN AND CARBON ISOTOPE RESULTS}

\section{Benthic Oxygen Isotopes}

Oxygen isotope data for Cibicidoides kullenbergi are plotted against depth in Figure 8, and against time in Figure 9 (Table 2). The data are discussed here within a chronological framework. During the last $6.6 \mathrm{Ma}$, the benthic oxygen isotope record exhibits a number of steps that represent changing average values over specific intervals. These steps are separated by relatively brief intervals of rapid change. Light oxygen isotope values $(1.80 \% 0$ avg.) are exhibited in the late Miocene between 6.4 and 5.6 Ma. A rapid enrichment in $\delta^{18} \mathrm{O}$ occurs at $5.6 \mathrm{Ma}$, within the latest Miocene, with relatively enriched values exhibited until $4.8 \mathrm{Ma}$ in the early Pliocene. This, in turn, is followed by a depletion in $\delta^{18} \mathrm{O}$ at $\sim 4.7 \mathrm{Ma}$, with lighter and more stable values marking the remaining early Pliocene. A gradual enrichment in $\delta^{18} \mathrm{O}\left(0.3 \%_{0}\right.$ avg. $)$ occurs between 3.7 and $3.4 \mathrm{Ma}$, at the end of the early Pliocene (centered at $3.6 \mathrm{Ma}$ ). A second permanent enrichment in $\delta^{18} \mathrm{O}(0.5 \%$ avg. $)$ occurs between 2.9 and $2.5 \mathrm{Ma}$, with heavy values marking the remainder of the Pliocene. 


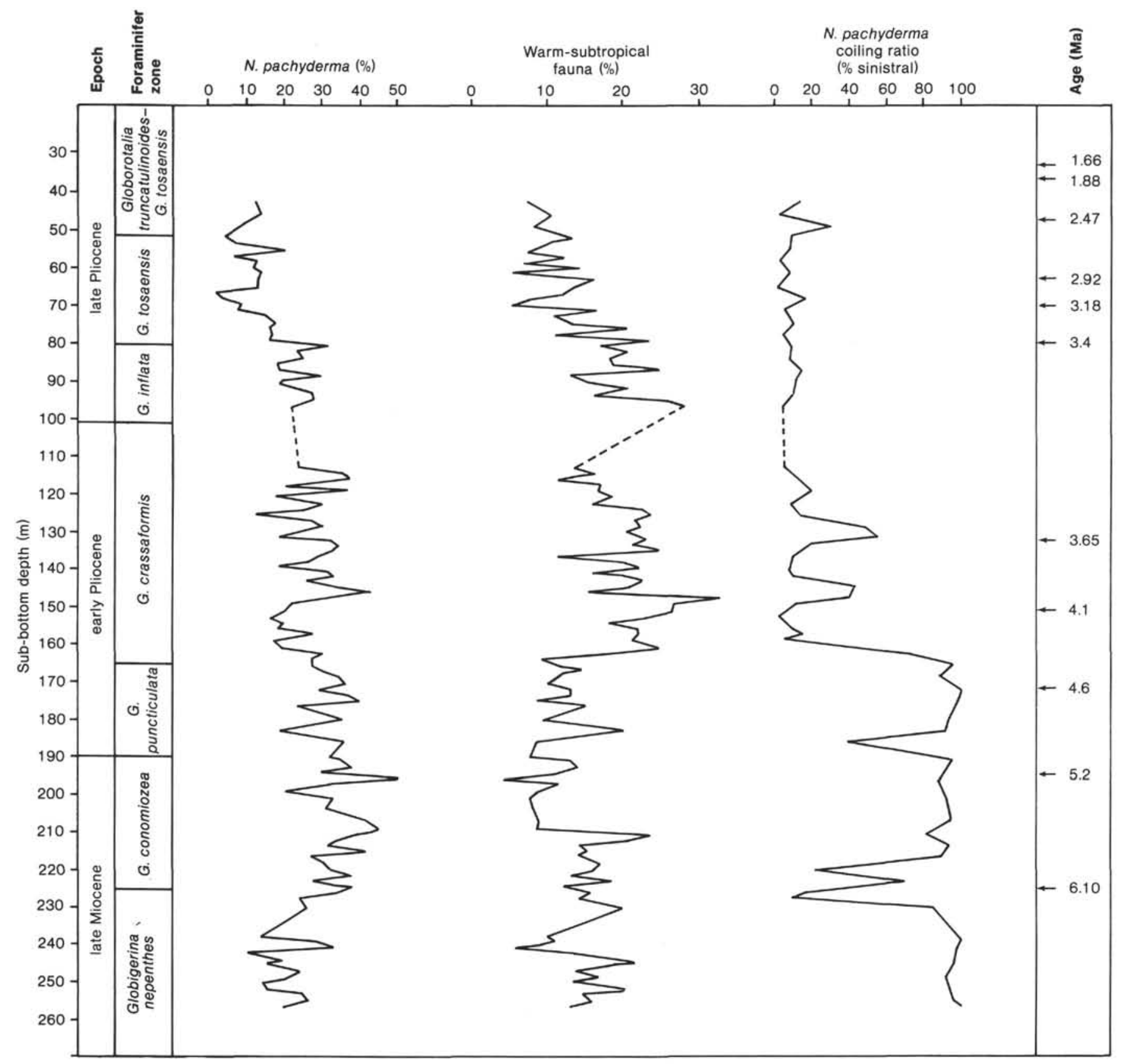

Figure 5. Frequency data for Neogloboquadrina pachyderma and warm-subtropical forms and the coiling ratio of $N$. pachyderma plotted against depth at Site 590.

\section{Planktonic Oxygen Isotopes}

The planktonic foraminiferal oxygen isotope data for Globigerinoides sacculifer are plotted against depth in Figure 8 and against time in Figure 9. The data indicate that the planktonic oxygen isotope record through the entire late Neogene is much more variable than the benthic oxygen isotope record. Such high variability would be expected in a site which is located close to a divergence zone that separates temperate from warm-subtropical waters and is associated with upwelling. The planktonic foraminiferal oxygen isotope record is quite different from the benthic record: it shows no obvious trends through time, and the average values in the latest Pliocene are no heavier than the average values exhibited in the latest Miocene, with average values not changing by more than $0.3 \%$ between 6.4 and $1.7 \mathrm{Ma}$. There are, however, slight average differences during various times. The late Miocene, between 6.3 and $5.5 \mathrm{Ma}$, is represented by average $\delta^{18} \mathrm{O}$ values of $\simeq 0.54 \%$, followed by a slight enrichment at $5.4 \mathrm{Ma}$ to average values of $\simeq 0.40 \%$ o until 3.7 Ma (a distinct brief lightening occurs between 4.9 and $4.6 \mathrm{Ma}$ ). Average values lighten to $\simeq 0.66 \%$ at 3.7 Ma, continuing until $3.1 \mathrm{Ma}$, when the oxygen isotope values again shift to slightly heavier values of $\simeq 0.57 \%$ for the remainder of the late Pliocene. 


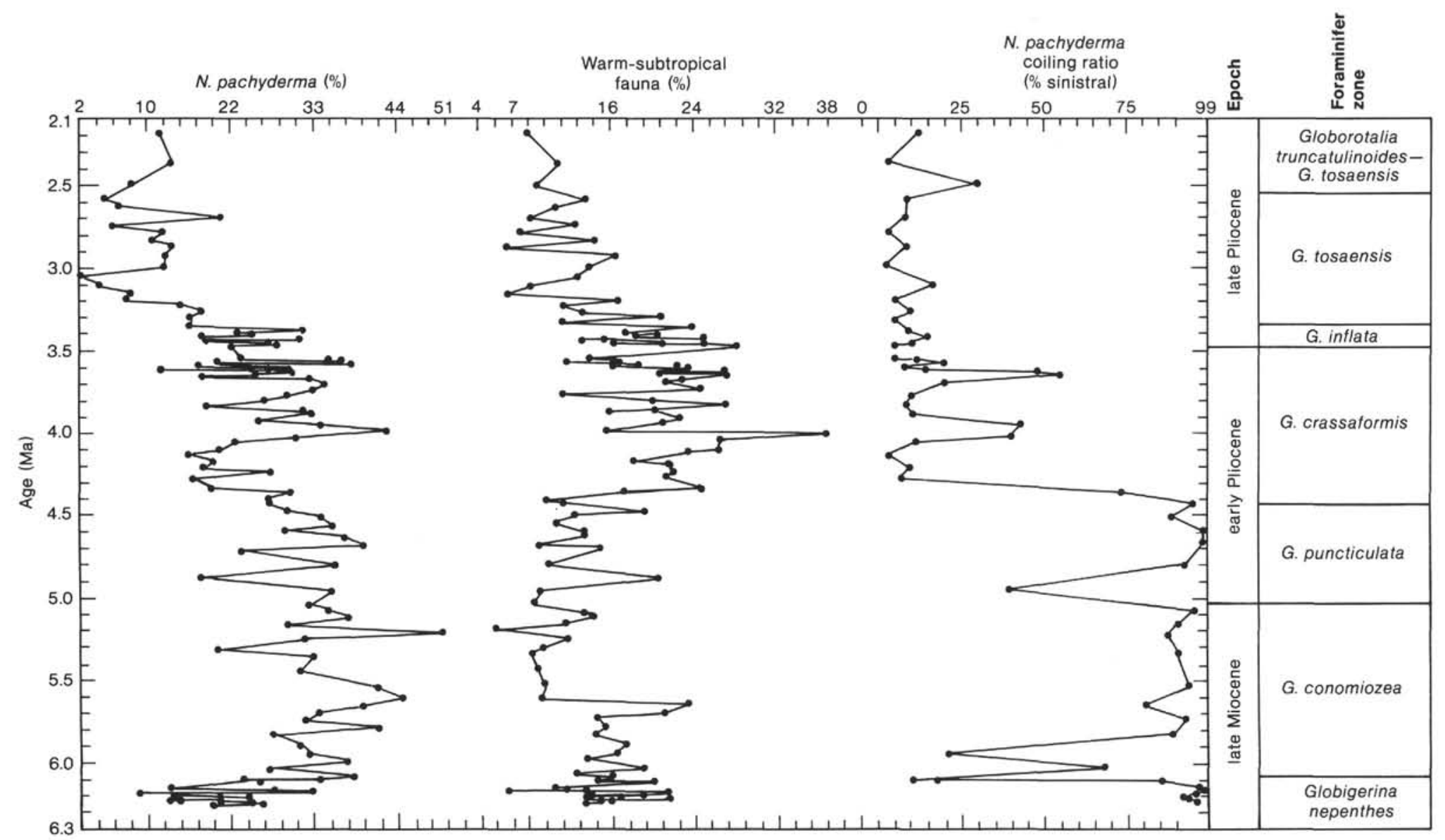

Figure 6. Frequency data for Neogloboquadrina pachyderma and warm-subtropical forms (\%) and the coiling ratio of $N$. pachyderma (\% sinistral) plotted against age at Site 590. 
Table 1. Distribution of planktonic foraminifers (frequency in \%) in Hole 590A.

\begin{tabular}{|c|c|c|c|c|c|c|c|c|c|c|c|c|c|c|c|c|c|c|c|c|c|c|c|c|c|c|c|c|c|c|c|c|c|c|c|c|c|}
\hline Epoch & $\begin{array}{l}\text { Foraminifer } \\
\text { zone }\end{array}$ & $\begin{array}{l}\text { Sub- } \\
\text { bottom } \\
\text { depth } \\
\text { (m) }\end{array}$ & $\begin{array}{l}\text { Core-Section } \\
\text { (level in } \mathrm{cm} \text { ) }\end{array}$ & 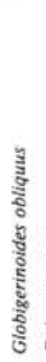 & 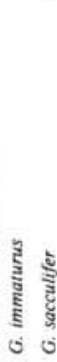 & 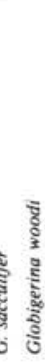 & & & & & & & & & o & & & & $\approx$ & & & 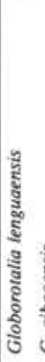 & 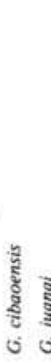 & & & & & $\begin{array}{ll} \\
\text { है } \\
0\end{array}$ & & & 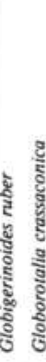 & & & & 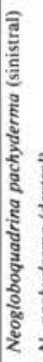 & 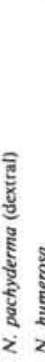 & \\
\hline \multirow{3}{*}{$\begin{array}{l}\text { late } \\
\text { Pliocene }\end{array}$} & $\begin{array}{l}\text { G. tosaensis- } \\
\text { G. Iruncatulinoides }\end{array}$ & $\begin{array}{l}42.2 \\
45.8 \\
48.8 \\
51.8\end{array}$ & $\begin{array}{l}2-5,35 \\
3-1,35 \\
3-3,35 \\
3-5,35\end{array}$ & $\begin{array}{l}2 \\
2 \\
4 \\
7\end{array}$ & $\begin{array}{ll}1 & x \\
x & 0 \\
1 & 1 \\
0 & 3\end{array}$ & $\begin{array}{lr}x & 7 \\
0 & 9 \\
1 & 7 \\
3 & 19\end{array}$ & $\begin{array}{l}0 \\
0 \\
0 \\
0\end{array}$ & & $\begin{array}{ll}0 & 0 \\
8 & 0 \\
7 & 0 \\
1 & 0\end{array}$ & $\begin{array}{l}16 \\
11\end{array}$ & $\begin{array}{l}0 \\
0 \\
0\end{array}$ & $\begin{array}{r}0 \\
0 \\
0 \\
18\end{array}$ & $\begin{array}{l}x \\
x \\
0 \\
1\end{array}$ & $\begin{array}{l}x \\
1 \\
0 \\
2\end{array}$ & $\begin{array}{ll}0 & 0 \\
0 & 0 \\
0 & 0 \\
0 & 0\end{array}$ & $\begin{array}{l}22 \\
0 \\
0 \\
0 \\
0 \\
0 \\
11\end{array}$ & & $\begin{array}{ll}x & 0 \\
2 & 0 \\
2 & 0 \\
x & 0\end{array}$ & $\begin{array}{l}6 \\
3 \\
5 \\
5 \\
4\end{array}$ & $\begin{array}{l}0 \\
0 \\
0 \\
0\end{array}$ & $\begin{array}{l}0 \\
0 \\
0 \\
0\end{array}$ & $\begin{array}{l}0 \\
0 \\
0 \\
0 \\
0\end{array}$ & $\begin{array}{ll}0 & 0 \\
0 & 0 \\
0 & 0 \\
0 & 0\end{array}$ & $\begin{array}{ll}0 & 0 \\
0 & 0 \\
0 & 0 \\
0 & 0\end{array}$ & $\begin{array}{ll}0 & x \\
0 & x \\
0 & x \\
0 & 0\end{array}$ & $\begin{array}{l}0 \\
0 \\
0 \\
0\end{array}$ & $\begin{array}{l}0 \\
0 \\
0 \\
0\end{array}$ & $\begin{array}{ll}4 & 0 \\
0 & 0 \\
x & 2 \\
2 & 2\end{array}$ & $\begin{array}{l}0 \\
0 \\
2 \\
2 \\
2\end{array}$ & $\begin{array}{l}0 \\
2 \\
6 \\
0\end{array}$ & & $\begin{array}{l}0 \\
0 \\
0 \\
0\end{array}$ & $\begin{array}{l}0 \\
0 \\
0 \\
0 \\
0\end{array}$ & $\begin{array}{l}12 \\
15 \\
24 \\
18\end{array}$ & & $\begin{array}{r}11 \\
14 \\
6 \\
4\end{array}$ & \\
\hline & G. tosaensis & $\begin{array}{l}53.3 \\
55.4 \\
56.9 \\
58.4 \\
59.9 \\
61.4 \\
62.9 \\
65.0 \\
66.5 \\
68.0 \\
69.5 \\
71.0 \\
72.5 \\
74.6 \\
76.1 \\
77.6 \\
79.1\end{array}$ & $\begin{array}{l}3-6,35 \\
4-1,35 \\
4-2,35 \\
4-3,35 \\
4-4,35 \\
4,5,35 \\
4-6,35 \\
5-1,110 \\
5-2,110 \\
5-3,110 \\
5-4,110 \\
5-5,110 \\
5-6,110 \\
6-1,55 \\
6-2,55 \\
6-3,55 \\
6-4,55\end{array}$ & $\begin{array}{l}4 \\
6 \\
7 \\
3 \\
8 \\
3 \\
6 \\
5 \\
5 \\
3 \\
3 \\
7 \\
6 \\
8 \\
7 \\
5 \\
6\end{array}$ & $\begin{array}{ll}0 & 2 \\
0 & 1 \\
3 & 2 \\
0 & 2 \\
0 & 2 \\
\mathrm{x} & \mathrm{x} \\
1 & 4 \\
\mathrm{x} & 3 \\
\mathrm{x} & 3 \\
0 & 2 \\
0 & \mathrm{x} \\
0 & 1 \\
0 & 1 \\
0 & 1 \\
0 & 1 \\
0 & 1 \\
0 & 1\end{array}$ & $\begin{array}{ll}2 & 21 \\
1 & 14 \\
2 & 10 \\
2 & 11 \\
2 & 11 \\
2 & 10 \\
4 & 11 \\
3 & 10 \\
3 & 10 \\
2 & 6 \\
x & 6 \\
1 & 8 \\
1 & 9 \\
1 & 8 \\
1 & 10 \\
1 & 16 \\
1 & 10 \\
1 & 6\end{array}$ & $\begin{array}{l}0 \\
0 \\
0 \\
0 \\
0 \\
0 \\
0 \\
0 \\
0 \\
0 \\
0 \\
0 \\
0 \\
0 \\
0 \\
0 \\
0\end{array}$ & & $\begin{array}{ll}7 & 0 \\
9 & 0 \\
9 & 0 \\
7 & 0 \\
2 & 0 \\
8 & 0 \\
5 & 0 \\
6 & 0 \\
0 & 0 \\
6 & 0 \\
9 & 0 \\
3 & 0 \\
5 & 0 \\
7 & 0 \\
4 & 0 \\
6 & 0 \\
5 & 0\end{array}$ & $\begin{array}{r}4 \\
4 \\
5 \\
3 \\
9 \\
5 \\
6 \\
9 \\
7 \\
8 \\
11\end{array}$ & $\begin{array}{l}0 \\
0 \\
0 \\
0 \\
0 \\
0 \\
0 \\
0 \\
0 \\
0 \\
\mathrm{x} \\
0 \\
0 \\
0 \\
0 \\
0 \\
0\end{array}$ & $\begin{array}{l}17 \\
17 \\
28 \\
29 \\
16 \\
24 \\
14 \\
22 \\
33 \\
36 \\
30 \\
21 \\
26 \\
23 \\
10 \\
18 \\
18 \\
17\end{array}$ & $\begin{array}{l}\mathrm{x} \\
\mathrm{x} \\
2 \\
\mathrm{x} \\
\mathrm{x} \\
2 \\
1 \\
1 \\
1 \\
1 \\
\mathrm{x} \\
2 \\
1 \\
\mathrm{x} \\
1 \\
\mathrm{x} \\
0 \\
2\end{array}$ & $\begin{array}{l}x \\
x \\
1 \\
x \\
4 \\
x \\
5 \\
3 \\
2 \\
1 \\
x \\
4 \\
2 \\
x \\
4 \\
2 \\
2\end{array}$ & $\begin{array}{ll}0 & 0 \\
0 & 0 \\
0 & x \\
0 & x \\
0 & 0 \\
2 & 0 \\
0 & 0 \\
1 & 0 \\
x & 0 \\
0 & 0 \\
0 & 0 \\
0 & 0 \\
0 & 0 \\
0 & 0 \\
0 & 0 \\
x & 0 \\
x & 0 \\
x & 2\end{array}$ & $\begin{array}{rr}0 & 17 \\
0 & 13 \\
\times & 18 \\
x & 15 \\
0 & 10 \\
0 & 17 \\
0 & 12 \\
0 & 12 \\
0 & 10 \\
0 & 14 \\
0 & 8 \\
x & 11 \\
0 & 8 \\
0 & 8 \\
0 & 10 \\
0 & 10 \\
2 & 11\end{array}$ & & $\begin{array}{ll}x & 0 \\
x & \\
x & \\
x & \\
1 & \\
2 & \\
3 & \\
1 & \\
x & \\
x & \\
x & \\
x & \\
x & \\
1 & \\
0 & \\
x & \\
x & \\
x & \\
0 & 13\end{array}$ & $\begin{array}{cc}0 & 5 \\
0 & 4 \\
0 & 4 \\
0 & 7 \\
0 & 6 \\
0 & 6 \\
x & 8 \\
x & 9 \\
1 & 10 \\
1 & 12 \\
1 & 7 \\
4 & 10 \\
1 & 8 \\
1 & 7 \\
9 & 7 \\
1 & 7 \\
3 & 4\end{array}$ & $\begin{array}{l}0 \\
x \\
0 \\
0 \\
0 \\
0 \\
0 \\
0 \\
x \\
x \\
2 \\
2 \\
0 \\
2 \\
1 \\
3 \\
0\end{array}$ & \begin{tabular}{l|}
0 \\
0 \\
0 \\
0 \\
0 \\
0 \\
0 \\
0 \\
0 \\
0 \\
0 \\
0 \\
0 \\
0 \\
0 \\
0 \\
0
\end{tabular} & 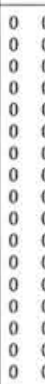 & $\begin{array}{ll}0 & 0 \\
0 & 0 \\
0 & 0 \\
0 & 0 \\
0 & 0 \\
0 & 0 \\
0 & 0 \\
0 & 0 \\
0 & 0 \\
0 & 0 \\
0 & 0 \\
0 & 0 \\
0 & 0 \\
0 & 0 \\
0 & 0 \\
0 & 0 \\
0 & 0 \\
0 & 0\end{array}$ & $\begin{array}{ll}0 & 0 \\
0 & 0 \\
0 & 0 \\
0 & 0 \\
0 & \mathrm{x} \\
0 & \mathrm{x} \\
0 & 0 \\
0 & 0 \\
0 & 0 \\
0 & 0 \\
0 & \mathrm{x} \\
0 & \mathrm{x} \\
0 & 1 \\
0 & 0 \\
0 & 0 \\
0 & 0 \\
0 & 0 \\
0 & 0\end{array}$ & $\begin{array}{ll}0 & 0 \\
0 & 0 \\
0 & 0 \\
0 & 0 \\
x & x \\
0 & 0 \\
0 \\
0 \\
0 \\
0 \\
0 \\
0 \\
0 \\
0 \\
0 \\
0 \\
0 \\
0 \\
0 \\
0 \\
0 \\
x\end{array}$ & $\begin{array}{l}0 \\
0 \\
0 \\
0 \\
0 \\
0 \\
0 \\
x \\
x \\
0 \\
0 \\
x \\
0 \\
0 \\
0 \\
0 \\
0 \\
x \\
0\end{array}$ & $\begin{array}{l}0 \\
0 \\
0 \\
0 \\
0 \\
0 \\
0 \\
0 \\
0 \\
1 \\
0 \\
0 \\
0 \\
0 \\
0 \\
0 \\
0\end{array}$ & $\begin{array}{lll}x & \\
4 & x \\
1 & 2 \\
3 & x \\
1 & 1 \\
3 & x \\
2 & 7 \\
2 & x \\
6 & 0 \\
2 & x \\
2 & 0 \\
6 & 0 \\
2 & 0 \\
x & 0 \\
3 & x \\
3 & 0 \\
4 & 0 & \\
4 & 0\end{array}$ & $\begin{array}{ll}4 & \\
x & \\
2 & \\
x & \\
1 & \\
x & \\
7 & \\
x & \\
0 & \\
x & \\
0 & \\
0 & \\
0 & \\
0 & \\
0 & \\
x & \\
0 & \\
0 & \\
0 & \end{array}$ & , & $\begin{array}{ll}3 & 4 \\
0 & 2 \\
1 & 2 \\
x & 2 \\
0 & 2 \\
x & x \\
0 & \mathrm{x} \\
0 & 0 \\
0 & \mathrm{x} \\
0 & 2 \\
0 & 3 \\
0 & \mathrm{x} \\
\mathrm{x} & 1 \\
2 & 1 \\
\mathrm{x} & 3 \\
\mathrm{x} & 3 \\
0 & 3 \\
& 2\end{array}$ & $\begin{array}{l}0 \\
0 \\
0 \\
0 \\
0 \\
0 \\
0 \\
0 \\
0 \\
0 \\
0 \\
0 \\
0 \\
0 \\
0 \\
0 \\
0\end{array}$ & & $\begin{array}{l}17 \\
17 \\
28 \\
29 \\
16 \\
24 \\
14 \\
22 \\
33 \\
36 \\
30 \\
21 \\
26 \\
23 \\
10 \\
18 \\
17\end{array}$ & $\begin{array}{l}3 \\
2 \\
0 \\
x \\
1 \\
1 \\
1 \\
x \\
0 \\
x \\
x \\
x \\
x \\
2 \\
2 \\
x \\
2 \\
2\end{array}$ & $\begin{array}{r}4 \\
19 \\
6 \\
12 \\
10 \\
13 \\
12 \\
13 \\
2 \\
3 \\
8 \\
7 \\
15 \\
16 \\
14 \\
16 \\
14\end{array}$ & $\begin{array}{l}\hat{0} \\
0 \\
0 \\
0 \\
2\end{array}$ \\
\hline & G. inflata & $\begin{array}{l}80.6 \\
82.1 \\
84.2 \\
85.7 \\
87.2 \\
88.7 \\
90.2 \\
91.7 \\
93.8 \\
95.3 \\
96.8\end{array}$ & $\begin{array}{l}6-5,55 \\
6-6,55 \\
7-1,35 \\
7-2,35 \\
7-3,35 \\
7-4,35 \\
7-5,36 \\
7-6,35 \\
8-1,103 \\
8-2,35 \\
8-3,35\end{array}$ & $\begin{array}{l}4 \\
9 \\
9 \\
7 \\
8 \\
7 \\
8 \\
5 \\
7 \\
6 \\
8\end{array}$ & $\begin{array}{rr}x & x \\
0 & 2 \\
0 & 3 \\
0 & 5 \\
0 & 8 \\
0 & 8 \\
0 & 3 \\
0 & 1 \\
0 & 11 \\
1 & 5 \\
4 & 11 \\
x & 4\end{array}$ & $\begin{array}{rr}x & 6 \\
2 & 8 \\
3 & 8 \\
5 & 14 \\
8 & 8 \\
3 & 8 \\
1 & 6 \\
1 & 6 \\
5 & 5 \\
1 & 10 \\
4 & 7 \\
4 & 8\end{array}$ & $\begin{array}{l}0 \\
0 \\
0 \\
0 \\
0 \\
0 \\
0 \\
0 \\
0 \\
0 \\
0\end{array}$ & & $\begin{array}{ll}5 & 0 \\
6 & 0 \\
5 & 0 \\
1 & 0 \\
8 & 0 \\
7 & 0 \\
4 & 0 \\
5 & 0 \\
9 & 0 \\
0 & 0 \\
9 & 0\end{array}$ & $\begin{array}{r}6 \\
9 \\
9 \\
11 \\
8 \\
8 \\
14 \\
14 \\
8 \\
7 \\
7\end{array}$ & $\begin{array}{l}0 \\
0 \\
x \\
0 \\
0 \\
x \\
x \\
0 \\
0 \\
x \\
0\end{array}$ & $\begin{array}{r}8 \\
7 \\
18 \\
9 \\
9 \\
8 \\
9 \\
13 \\
9 \\
4 \\
8\end{array}$ & $\begin{array}{l}2 \\
x \\
1 \\
1 \\
1 \\
x \\
x \\
0 \\
1 \\
1 \\
x\end{array}$ & $\begin{array}{l}2 \\
3 \\
2 \\
4 \\
2 \\
1 \\
2 \\
4 \\
2 \\
1 \\
4\end{array}$ & $\begin{array}{ll}2 & x \\
x & 0 \\
x & 1 \\
x & 0 \\
9 & 8 \\
x & 0 \\
x & 0 \\
x & 0 \\
x & 0 \\
2 & x \\
6 & 1\end{array}$ & $\begin{array}{ll}x & 11 \\
0 & 11 \\
1 & 5 \\
0 & 9 \\
8 & 9 \\
0 & 9 \\
0 & 7 \\
0 & 9 \\
0 & 9 \\
x & 0 \\
1 & 9\end{array}$ & $\mid \begin{array}{l}x \\
0 \\
x \\
x \\
x \\
x \\
x \\
1 \\
x \\
x \\
0\end{array}$ & $\begin{array}{ll}x & \\
0 & \\
x & 2 \\
x & 1 \\
x & 6 \\
x & 1 \\
x & \\
1 & \\
x & 1 \\
x & \\
0 & \\
0 & 3\end{array}$ & $\begin{array}{ll}7 & 5 \\
4 & 6 \\
2 & 5 \\
1 & 6 \\
6 & 6 \\
1 & 4 \\
4 & 7 \\
1 & 8 \\
1 & 5 \\
x & 4 \\
3 & 4\end{array}$ & $\begin{array}{l}2 \\
1 \\
x \\
x \\
x \\
0 \\
0 \\
x \\
x \\
x \\
0 \\
0\end{array}$ & $\begin{array}{l}0 \\
0 \\
0 \\
0 \\
0 \\
0 \\
0 \\
0 \\
0 \\
0 \\
0\end{array}$ & $\begin{array}{ll}0 & \\
0 & \\
0 & \\
0 & \\
0 & \\
0 & \\
0 & \\
0 & \\
0 & \\
0 & \\
0 & \\
0 & \end{array}$ & $\begin{array}{ll}0 & 0 \\
0 & 0 \\
0 & 0 \\
0 & 0 \\
0 & 0 \\
0 & 0 \\
0 & 0 \\
0 & 0 \\
0 & 0 \\
0 & 0 \\
0 & 0 \\
0 & 0\end{array}$ & $\begin{array}{ll}0 & 0 \\
0 & 0 \\
0 & 0 \\
0 & 0 \\
0 & 0 \\
0 & 0 \\
0 & 0 \\
0 & 0 \\
0 & 0 \\
0 & 0 \\
0 & 0 \\
0 & 0\end{array}$ & $\begin{array}{l}x \\
0 \\
0 \\
x \\
0 \\
0 \\
x \\
0 \\
0 \\
0 \\
x \\
0 \\
x \\
x\end{array}$ & $\begin{array}{l}\mathrm{x} \\
2 \\
0 \\
\mathrm{x} \\
1 \\
0 \\
1 \\
3 \\
0 \\
\mathrm{x} \\
\mathrm{x} \\
\end{array}$ & $\begin{array}{l}0 \\
\mathrm{x} \\
0 \\
0 \\
\mathrm{x} \\
0 \\
0 \\
0 \\
0 \\
0 \\
0\end{array}$ & $\begin{array}{ll}6 & 0 \\
x & 0 \\
2 & 0 \\
0 & 0 \\
1 & 0 \\
4 & 0 \\
x & 0 \\
0 & 0 \\
3 & 0 \\
1 & 0 \\
1 & 0 \\
1\end{array}$ & 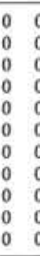 & 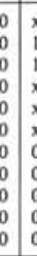 & $\begin{array}{ll}x & x \\
1 & 1 \\
1 & 1 \\
x & 0 \\
x & 1 \\
x & 4 \\
0 & 5 \\
0 & 1 \\
0 & x \\
0 & 1 \\
0 & 1\end{array}$ & $\begin{array}{l}0 \\
0 \\
0 \\
0 \\
0 \\
0 \\
0 \\
0 \\
0 \\
0 \\
0\end{array}$ & $\begin{array}{l}0 \\
0 \\
0 \\
0 \\
0 \\
0 \\
0 \\
0 \\
0 \\
0 \\
0\end{array}$ & \begin{tabular}{r|r}
8 \\
7 \\
18 \\
9 \\
9 \\
8 \\
9 \\
13 \\
0 \\
0 \\
0
\end{tabular} & & $\begin{array}{l}29 \\
20 \\
23 \\
17 \\
16 \\
30 \\
17 \\
16 \\
24 \\
24 \\
21\end{array}$ & $\begin{array}{l}0 \\
0 \\
0 \\
0 \\
0 \\
0 \\
x \\
1\end{array}$ \\
\hline \multirow[t]{2}{*}{$\begin{array}{l}\text { early } \\
\text { Pliocene }\end{array}$} & G. crassaformis & $\begin{array}{l}113.0 \\
114.5 \\
116.0 \\
118.0 \\
119.0 \\
120.5 \\
122.6 \\
124.1 \\
125.6 \\
127.1 \\
128.6 \\
130.1 \\
131.6 \\
132.2 \\
133.7 \\
135.2 \\
136.7 \\
138.2 \\
139.7 \\
141.2 \\
141.8 \\
143.3 \\
144.8 \\
146.3 \\
147.8 \\
149.3 \\
151.4 \\
152.9 \\
154.4 \\
155.9 \\
157.4 \\
158.9 \\
161.0 \\
162.5 \\
164.0 \\
165.5\end{array}$ & 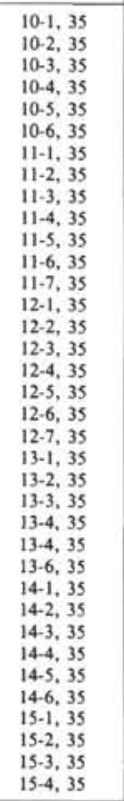 & $\begin{array}{r}7 \\
6 \\
4 \\
5 \\
7 \\
6 \\
2 \\
9 \\
9 \\
10 \\
8 \\
3 \\
9 \\
8 \\
7 \\
5 \\
4 \\
7 \\
8 \\
6 \\
14 \\
14 \\
12 \\
8 \\
10 \\
10 \\
12 \\
11 \\
12 \\
10 \\
7 \\
8 \\
14 \\
10 \\
4 \\
7 \\
\end{array}$ & $\begin{array}{ll}0 & 2 \\
0 & 2 \\
0 & 1 \\
0 & 0 \\
0 & \mathrm{x} \\
0 & 6 \\
0 & 3 \\
0 & 5 \\
0 & 2 \\
0 & 5 \\
0 & 4 \\
\mathrm{x} & 2 \\
0 & 4 \\
0 & 3 \\
0 & 4 \\
0 & 4 \\
0 & 1 \\
0 & 1 \\
\mathrm{x} & 5 \\
0 & 3 \\
\mathrm{x} & \mathrm{x} \\
0 & 3 \\
0 & 2 \\
0 & 2 \\
0 & 7 \\
0 & 4 \\
\mathrm{x} & 5 \\
0 & 3 \\
0 & 2 \\
0 & 7 \\
\mathrm{x} & 3 \\
1 & 4 \\
0 & 5 \\
1 & 2 \\
\mathrm{x} & 2 \\
0 & \mathrm{x}\end{array}$ & $\begin{array}{lc}2 & 8 \\
2 & 9 \\
1 & 7 \\
0 & 12 \\
x & 7 \\
6 & 9 \\
3 & 7 \\
5 & 15 \\
2 & 9 \\
5 & 4 \\
4 & 7 \\
2 & 9 \\
4 & 91 \\
3 & 11 \\
4 & 8 \\
4 & 13 \\
1 & 9 \\
1 & 15 \\
5 & 10 \\
3 & 10 \\
x & 13 \\
3 & 11 \\
2 & 13 \\
2 & 10 \\
7 & 10 \\
4 & 15 \\
5 & 15 \\
3 & 13 \\
2 & 17 \\
7 & 10 \\
3 & 13 \\
4 & 13 \\
5 & 13 \\
2 & 11 \\
2 & 11 \\
2 & 11 \\
x & 20\end{array}$ & $\begin{array}{l}0 \\
x \\
0 \\
0 \\
0 \\
0 \\
0 \\
x \\
0 \\
0 \\
0 \\
0 \\
0 \\
0 \\
0 \\
0 \\
0 \\
x \\
0 \\
0 \\
0 \\
0 \\
0 \\
0 \\
0 \\
0 \\
0 \\
0 \\
0 \\
0 \\
0 \\
0 \\
0 \\
x \\
0 \\
0\end{array}$ & $\begin{array}{r}11 \\
7 \\
11 \\
8 \\
4 \\
7 \\
3 \\
9 \\
9 \\
13 \\
7 \\
14 \\
19 \\
10 \\
6 \\
8 \\
13 \\
12 \\
9 \\
14 \\
13 \\
7 \\
6 \\
5 \\
5 \\
2 \\
12 \\
10 \\
5 \\
7 \\
7 \\
9 \\
9 \\
8 \\
18 \\
11\end{array}$ & 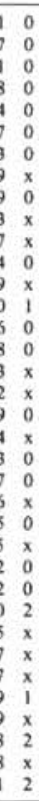 & $\begin{array}{r}7 \\
10 \\
8 \\
11 \\
7 \\
18 \\
14 \\
7 \\
3 \\
4 \\
10 \\
5 \\
7 \\
8 \\
9 \\
5 \\
9 \\
8 \\
12 \\
7 \\
4 \\
9 \\
9 \\
8 \\
x \\
5 \\
8 \\
8 \\
14 \\
12 \\
6 \\
10 \\
9 \\
4 \\
0\end{array}$ & $\begin{array}{l}0 \\
x \\
0 \\
0 \\
x \\
x \\
0 \\
x \\
x \\
2 \\
x \\
2 \\
x \\
1 \\
x \\
x \\
x \\
2 \\
3 \\
x \\
2 \\
2 \\
3 \\
1 \\
1 \\
2 \\
3 \\
3 \\
2 \\
6 \\
5 \\
6 \\
3 \\
4 \\
2 \\
2\end{array}$ & $\begin{aligned} 13 \\
5 \\
2 \\
19 \\
11 \\
14 \\
11 \\
0 \\
23 \\
6 \\
5 \\
14 \\
11 \\
2 \\
5 \\
5 \\
13 \\
2 \\
2 \\
13 \\
4 \\
7 \\
8 \\
x \\
1 \\
2 \\
1 \\
6 \\
4 \\
4 \\
2 \\
x \\
x \\
2 \\
2 \\
2 \\
4 \\
3 \\
2 \\
\end{aligned}$ & $\begin{array}{l}2 \\
2 \\
2 \\
x \\
x \\
0 \\
x \\
x \\
1 \\
1 \\
x \\
2 \\
0 \\
0 \\
3 \\
x \\
x \\
x \\
2 \\
x \\
x \\
x \\
x \\
x \\
x \\
x \\
3 \\
x \\
1 \\
2 \\
4 \\
x \\
5 \\
x \\
x \\
0 \\
x \\
x \\
\end{array}$ & 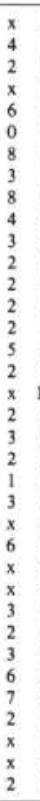 & $\begin{array}{ll}3 & x \\
1 & 2 \\
2 & 2 \\
8 & x \\
x & x \\
6 & x \\
x & 2 \\
1 & 4 \\
4 & 0 \\
2 & 0 \\
2 & 4 \\
8 & 4 \\
6 & 5 \\
5 & 5 \\
4 & 3 \\
9 & 1 \\
0 & 2 \\
10 & x \\
0 & 4 \\
0 & 3 \\
0 & 2 \\
2 & \mathrm{x} \\
0 & 2 \\
x & 2 \\
0 & 3 \\
8 & 3 \\
6 & \mathrm{x} \\
x & 4 \\
0 & 2 \\
0 & 2 \\
\mathrm{x} & 2 \\
0 & \mathrm{x} \\
0 & 2 \\
0 & \mathrm{x} \\
0 & \mathrm{x} \\
0 & \mathrm{x} \\
\end{array}$ & $\begin{array}{ll}x & 11 \\
2 & 4 \\
2 & 7 \\
x & 8 \\
x & 7 \\
x & 8 \\
2 & 8 \\
4 & 7 \\
0 & 4 \\
0 & 8 \\
4 & 8 \\
4 & 3 \\
5 & 3 \\
5 & 3 \\
3 & 3 \\
1 & 4 \\
2 & 4 \\
x & 2 \\
4 & 3 \\
3 & 4 \\
2 & 2 \\
x & 4 \\
2 & 2 \\
2 & 6 \\
3 & 4 \\
0 & 8 \\
x & 6 \\
4 & 4 \\
2 & 4 \\
2 & 7 \\
2 & 6 \\
x & 7 \\
2 & 2 \\
x & 5 \\
x & 6 \\
1 & 3 \\
\end{array}$ & $\begin{array}{l}1 \\
x \\
x \\
x \\
2 \\
x \\
2 \\
1 \\
1 \\
x \\
x \\
0 \\
2 \\
2 \\
1 \\
1 \\
4 \\
2 \\
0 \\
2 \\
4 \\
2 \\
x \\
x \\
1 \\
3 \\
1 \\
1 \\
2 \\
1 \\
x \\
1 \\
2 \\
1 \\
3\end{array}$ & $\begin{array}{ll}1 & x \\
x & 2 \\
x & 1 \\
x & x \\
2 & 1 \\
x & x \\
2 & 0 \\
1 & x \\
1 & x \\
x & 1 \\
x & 2 \\
0 & x \\
2 & x \\
2 & x \\
1 & 1 \\
1 & x \\
4 & x \\
2 & x \\
0 & 3 \\
2 & x \\
4 & \\
2 & 2 \\
x & 3 \\
x & 2 \\
1 & 7 \\
3 & 4 \\
1 & 2 \\
1 & 2 \\
2 & x \\
1 & 0 \\
x & 3 \\
1 & 0 \\
2 & 2 \\
1 & 3 \\
3 & x \\
5 & 0 \\
\end{array}$ & $\begin{array}{ll}x & 4 \\
2 & 2 \\
1 & 4 \\
x & 1 \\
1 & 3 \\
x & 2 \\
0 & 1 \\
x & 3 \\
x & \mathrm{x} \\
1 & 8 \\
2 & 8 \\
x & \mathrm{x} \\
x & 2 \\
x & 2 \\
1 & 2 \\
x & 2 \\
x & 2 \\
x & 1 \\
3 & 4 \\
x & 4 \\
1 & 1 \\
2 & \mathrm{x} \\
3 & 2 \\
2 & 2 \\
7 & 2 \\
4 & 3 \\
2 & \mathrm{x} \\
2 & 2 \\
x & 1 \\
0 & 2 \\
3 & 5 \\
0 & 5 \\
2 & 3 \\
3 & 3 \\
x & 2 \\
0 & 3 \\
0\end{array}$ & $\begin{array}{l}3 \\
2 \\
x \\
x \\
0 \\
x \\
x \\
1 \\
0 \\
0 \\
0 \\
x \\
x \\
1 \\
x \\
x \\
0 \\
0 \\
2 \\
x \\
0 \\
0 \\
x \\
0 \\
0 \\
0 \\
0 \\
0 \\
0 \\
x \\
x \\
x \\
0 \\
0 \\
2 \\
2 \\
4 \\
x\end{array}$ & \begin{tabular}{l|}
0 \\
0 \\
0 \\
0 \\
0 \\
0 \\
0 \\
0 \\
0 \\
0 \\
0 \\
0 \\
0 \\
0 \\
0 \\
0 \\
0 \\
0 \\
0 \\
0 \\
0 \\
0 \\
0 \\
0 \\
0 \\
0 \\
0 \\
0 \\
0 \\
0 \\
0 \\
0 \\
0 \\
0 \\
0
\end{tabular} & 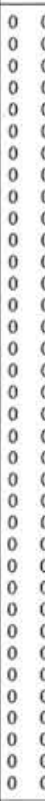 & $\begin{array}{ll}0 & 0 \\
0 & 0 \\
0 & 0 \\
0 & 0 \\
0 & 0 \\
0 & 0 \\
0 & 0 \\
0 & 0 \\
0 & 0 \\
0 & 0 \\
0 & 0 \\
0 & 0 \\
0 & 0 \\
0 & 0 \\
0 & 0 \\
0 & 0 \\
0 & 0 \\
0 & 0 \\
0 & 0 \\
0 & 0 \\
0 & 0 \\
0 & 0 \\
0 & 0 \\
0 & 0 \\
0 & 0 \\
0 & 0 \\
0 & 0 \\
0 & 0 \\
0 & 0 \\
0 & 0 \\
0 & 0 \\
0 & 0 \\
0 & 0 \\
0 & 0 \\
0 & 0 \\
0 & 0 \\
\end{array}$ & $\begin{array}{ll}0 & 0 \\
0 & 0 \\
0 & 0 \\
0 & 0 \\
0 & 0 \\
0 & 0 \\
0 & 0 \\
0 & 0 \\
0 & 0 \\
0 & 0 \\
0 & 0 \\
0 & 0 \\
0 & 0 \\
0 & 0 \\
0 & 0 \\
0 & 0 \\
0 & 0 \\
0 & \mathrm{x} \\
0 & 0 \\
0 & \mathrm{x} \\
0 & 0 \\
0 & 0 \\
0 & 2 \\
0 & 0 \\
0 & 0 \\
0 & 0 \\
0 & 0 \\
0 & 0 \\
0 & 0 \\
0 & 0 \\
0 & 0 \\
0 & 0 \\
0 & 0 \\
0 & 0 \\
0 & 0 \\
0 & \mathrm{x} \\
0 & 0 \\
0 & 0 \\
\end{array}$ & $\begin{array}{ll} & x \\
0 & 0 \\
0 \\
0 \\
0 \\
0 \\
0 \\
0 \\
0 \\
0 \\
0 \\
0 \\
0 \\
0 \\
0 \\
0 \\
0 \\
0 \\
1 \\
0 \\
0 \\
0 \\
0 \\
0 \\
0 \\
x \\
0 \\
0 \\
0 \\
x \\
x \\
x \\
x \\
x \\
0 \\
x \\
x \\
x \\
0 \\
x \\
x \\
0 \\
0 \\
x \\
x \\
x \\
x\end{array}$ & \begin{tabular}{|l}
$\mathrm{x}$ \\
0 \\
$\mathrm{x}$ \\
0 \\
0 \\
0 \\
0 \\
0 \\
$\mathrm{x}$ \\
0 \\
0 \\
$\mathrm{x}$ \\
0 \\
0 \\
0 \\
0 \\
0 \\
1 \\
2 \\
$\mathrm{x}$ \\
$\mathrm{x}$ \\
$\mathrm{x}$ \\
$\mathrm{x}$ \\
$\mathrm{x}$ \\
1 \\
$\mathrm{x}$ \\
0 \\
0 \\
2 \\
2 \\
$\mathrm{x}$ \\
0 \\
1 \\
1 \\
\\
3 \\
$\mathrm{x}$ \\
$\mathrm{x}$ \\
$\mathrm{x}$ \\
\end{tabular} & $\begin{aligned} 0 \\
0 \\
\mathrm{x} \\
\mathrm{x} \\
0 \\
0 \\
0 \\
0 \\
\mathrm{x} \\
2 \\
2 \\
0 \\
3 \\
\mathrm{x} \\
3 \\
3 \\
5 \\
\mathrm{x} \\
\mathrm{x} \\
5 \\
0 \\
5 \\
0 \\
4 \\
4 \\
5 \\
4 \\
4 \\
7 \\
3 \\
3 \\
9 \\
7 \\
10 \\
2 \\
\mathrm{x} \\
8 \\
6 \\
5 \\
5 \\
4\end{aligned}$ & $\begin{array}{rll}4 & 0 \\
5 & 0 \\
8 & 0 \\
2 & 0 \\
x & 0 \\
4 & 0 \\
5 & 0 \\
8 & 0 \\
10 & 0 \\
7 & 0 \\
8 & 0 \\
x & 0 \\
x & 0 \\
1 & 0 \\
1 & 0 \\
x & 0 \\
x & 0 \\
0 & 0 \\
0 & 0 \\
0 & 0 \\
0 & 0 \\
x & 0 \\
x & 0 \\
0 & 0 \\
0 & 0 \\
0 & 0 \\
0 & 0 \\
0 & 0 \\
0 & 0 \\
0 & 0 \\
x & 0 \\
x & 0 \\
x & 0 \\
x & 0 \\
x & 0 \\
x & 0 \\
\end{array}$ & $\begin{array}{l}0 \\
0 \\
0 \\
0 \\
0 \\
0 \\
0 \\
0 \\
0 \\
0 \\
0 \\
0 \\
0 \\
0 \\
0 \\
0 \\
0 \\
0 \\
0 \\
0 \\
0\end{array}$ & $\begin{array}{c}0 \\
0 \\
0 \\
0 \\
0 \\
0 \\
0 \\
0 \\
0 \\
0 \\
0 \\
0 \\
0 \\
0 \\
0 \\
0 \\
0 \\
0 \\
0\end{array}$ & $\begin{array}{ll}0 & 0 \\
0 & 0 \\
0 & 0 \\
0 & 0 \\
0 & 0 \\
0 & 0 \\
0 & 0 \\
0 & 0 \\
0 & 0 \\
0 & 0 \\
0 & 0 \\
0 & 0 \\
0 & 0 \\
0 & 0 \\
0 & 0 \\
0 & 0 \\
0 & 0 \\
0 & 0 \\
0 & 0 \\
0 & 0 \\
0 & 0 \\
0 & 0 \\
0 & 0 \\
0 & 0 \\
0 & 0 \\
0 & 0 \\
0 & 0 \\
0 & 0 \\
0 & 0 \\
0 & 0 \\
x & 0 \\
0 & 0 \\
0 & 0 \\
0 & 0 \\
0 & 0 \\
0 & 0 \\
0 & 0 \\
\end{array}$ & $\begin{array}{l}0 \\
0 \\
0 \\
0 \\
0 \\
0 \\
0 \\
0 \\
0 \\
0 \\
0 \\
0 \\
0 \\
0 \\
0 \\
0 \\
0 \\
0 \\
0 \\
0 \\
0 \\
0 \\
0 \\
0 \\
0\end{array}$ & $\begin{array}{ll}0 & 0 \\
0 & 0 \\
0 & 0 \\
0 & 0 \\
0 & 0 \\
0 & 0 \\
0 & 0 \\
0 & 0 \\
0 & 0 \\
0 & 0 \\
0 & 0 \\
0 & 0 \\
0 & 0 \\
0 & 0 \\
0 & 0 \\
0 & 0 \\
0 & 0 \\
0 & 0 \\
0 & 0 \\
0 & 0 \\
0 & 0 \\
0 & 0 \\
0 & 0 \\
0 & 0 \\
0 & 0 \\
0 & 0 \\
0 & 0 \\
0 & 0 \\
0 & 0 \\
0 & 0 \\
0 & 0 \\
0 & 0 \\
0 & 0 \\
0 & 0 \\
0 & 0 \\
0 & 0\end{array}$ & \begin{tabular}{l|l}
0 \\
0 \\
0 \\
0 \\
0 \\
0 \\
0 \\
0 \\
0 \\
0 \\
0 \\
0 \\
0 \\
0 \\
0 \\
0 \\
0 \\
0 \\
0 \\
0 \\
0 \\
0 \\
0 \\
0 \\
0 \\
0 \\
0 \\
0 \\
0 \\
0 \\
0 \\
0 \\
0 \\
0
\end{tabular} & $\begin{array}{cc}1 & 2 \\
2 & 3 \\
4 & 3 \\
12 \\
8 \\
1 \\
3 \\
3 \\
2 \\
2 \\
x \\
15 \\
6 \\
10 \\
12 \\
7 \\
1 \\
3 \\
3 \\
2 \\
2 \\
14 \\
14 \\
3 \\
10 \\
15 \\
21 \\
12 \\
3 \\
3 \\
x \\
x \\
x \\
2 \\
2 \\
4 \\
4 \\
1 & 2 \\
2 & 1 \\
22 & 2 \\
23 \\
26\end{array}$ & $\begin{aligned} 22 \\
33 \\
33 \\
8 \\
31 \\
16 \\
27 \\
22 \\
11 \\
27 \\
16 \\
19 \\
8 \\
21 \\
28 \\
32 \\
26 \\
24 \\
17 \\
18 \\
30 \\
16 \\
19 \\
22 \\
18 \\
20 \\
19 \\
16 \\
18 \\
16 \\
23 \\
16 \\
17 \\
8 \\
4 \\
1\end{aligned}$ & $\begin{array}{l}x \\
4 \\
3 \\
2 \\
x \\
2 \\
2 \\
2 \\
3 \\
0 \\
x \\
4 \\
4 \\
2 \\
2 \\
2 \\
x \\
2 \\
2\end{array}$ \\
\hline & G. puncticulata & $\begin{array}{l}167.0 \\
168.5 \\
170.6 \\
172.1 \\
173.6 \\
175.1 \\
176.6 \\
180.2 \\
183.2 \\
186.2 \\
189.8 \\
\end{array}$ & $\begin{array}{l}15-5,35 \\
15-6,35 \\
16-1,35 \\
16-2,35 \\
16-3.35 \\
16-4,35 \\
16-5,35 \\
17-1,35 \\
17-3,35 \\
17-5,35 \\
18-1,35\end{array}$ & $\begin{array}{r}8 \\
3 \\
3 \\
6 \\
6 \\
3 \\
8 \\
8 \\
6 \\
11 \\
5 \\
4\end{array}$ & $\begin{array}{ll}\mathrm{x} & 1 \\
2 & 2 \\
\mathrm{x} & 3 \\
\mathrm{x} & 3 \\
\mathrm{x} & 2 \\
\mathrm{x} & \mathrm{x} \\
2 & 2 \\
\mathrm{x} & 2 \\
\mathrm{x} & 4 \\
0 & 2 \\
\mathrm{x} & 2\end{array}$ & $\begin{array}{ll}1 & 14 \\
2 & 15 \\
3 & 8 \\
3 & 8 \\
2 & 13 \\
2 & 16 \\
2 & 16 \\
2 & 11 \\
2 & 12 \\
4 & 18 \\
2 & 11 \\
2 & 6\end{array}$ & $\begin{array}{l}x \\
0 \\
0 \\
0 \\
0 \\
0 \\
0 \\
0 \\
0\end{array}$ & & $\begin{array}{l}3 x \\
y \\
y \\
x \\
2 \\
2 x \\
x \\
x \\
1 \\
x \\
x \\
x \\
x \\
x\end{array}$ & $\begin{array}{l}3 \\
5 \\
9 \\
8 \\
4 \\
7 \\
\times \\
7 \\
5\end{array}$ & $\begin{array}{l}4 \\
4 \\
3 \\
2 \\
5 \\
3 \\
5\end{array}$ & $\begin{array}{l}2 \\
x \\
2 \\
x \\
x \\
2 \\
1 \\
0 \\
0 \\
x \\
9 \\
9\end{array}$ & $\begin{array}{l}x \\
x \\
x \\
x \\
0 \\
x\end{array}$ & $\begin{array}{l}5 \\
1 \\
0 \\
0 \\
0 \\
0 \\
0 \\
x \\
4 \\
4\end{array}$ & $\begin{array}{ll}0 & 1 \\
0 & 1 \\
0 & 3 \\
3 & \mathrm{x} \\
5 & \mathrm{x} \\
5 & 0 \\
\mathrm{x} & 0 \\
0 & 0 \\
0 & 0 \\
1 & 0 \\
1 & 0 \\
1 & 0\end{array}$ & $\begin{array}{l}1 \\
1 \\
3 \\
x \\
x \\
0 \\
0 \\
0 \\
0\end{array}$ & & & $\begin{array}{ll}2 & 5 \\
3 & 3 \\
x & 4 \\
0 & 6 \\
0 & 2 \\
0 & 2 \\
2 & 5 \\
0 & 5 \\
1 & 0 \\
1 & 3 \\
0 & 1 \\
0 & x\end{array}$ & $\begin{array}{l}x \\
x \\
x \\
0 \\
0 \\
0 \\
0 \\
x \\
x \\
0\end{array}$ & $\begin{array}{l}2 \\
0 \\
0 \\
0 \\
0 \\
0 \\
3 \\
\times \\
x \\
x \\
0\end{array}$ & 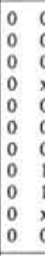 & $\begin{array}{ll}0 & 0 \\
0 & 0 \\
0 & 0 \\
x & 0 \\
0 & 0 \\
0 & 0 \\
0 & 0 \\
1 & 0 \\
1 & 0 \\
x & 0 \\
0 & 0 \\
\end{array}$ & $\begin{array}{ll}0 & 0 \\
0 & 0 \\
0 & 0 \\
0 & 0 \\
0 & 0 \\
0 & 0 \\
0 & 0 \\
0 & 0 \\
0 & 0 \\
0 & 0 \\
0 & 0 \\
\end{array}$ & $\begin{array}{l}0 \\
0 \\
0 \\
x \\
0 \\
0 \\
x \\
0 \\
x \\
1 \\
0\end{array}$ & \begin{tabular}{|l|}
0 \\
$x$ \\
1 \\
2 \\
2 \\
2 \\
0 \\
0 \\
0 \\
0 \\
0 \\
0 \\
\end{tabular} & $\begin{array}{r}5 \\
8 \\
7 \\
5 \\
4 \\
0 \\
12 \\
5 \\
8 \\
9 \\
x \\
\end{array}$ & $\begin{array}{ll}0 & 0 \\
0 & 0 \\
0 & 0 \\
0 & 0 \\
0 & 0 \\
0 & 0 \\
0 & 0 \\
0 & 0 \\
0 & 0 \\
0 & 0 \\
0 & 0 \\
\end{array}$ & $\begin{array}{l}0 \\
0 \\
0 \\
0 \\
0 \\
0 \\
0 \\
0 \\
0 \\
\end{array}$ & & $\begin{array}{ll}0 & 0 \\
0 & 0 \\
0 & 0 \\
0 & 0 \\
0 & 0 \\
0 & 0 \\
0 & 0 \\
0 & 0 \\
0 & 0 \\
0 & 0 \\
0 & 0 \\
\end{array}$ & & $\begin{array}{l}0 \\
0 \\
0 \\
0 \\
0 \\
0 \\
0 \\
0 \\
0 \\
0 \\
0 \\
0 \\
0 \\
0\end{array}$ & $\begin{array}{l}0 \\
0 \\
0 \\
0 \\
0 \\
0 \\
0 \\
0 \\
0 \\
0 \\
0\end{array}$ & $\begin{array}{l}28 \\
30 \\
34 \\
29 \\
36 \\
39 \\
23 \\
33 \\
16 \\
15 \\
25 \\
25\end{array}$ & $\begin{array}{r}1 \\
4 \\
2 \\
x \\
1 \\
x \\
x \\
3 \\
2 \\
23\end{array}$ & $\begin{array}{l}0 \\
0 \\
0 \\
0\end{array}$ \\
\hline $\begin{array}{l}\text { late } \\
\text { Miocene }\end{array}$ & G. conomiozea & $\begin{array}{l}191.3 \\
192.8 \\
194.3\end{array}$ & $\begin{array}{l}18-2,35 \\
18-3,35 \\
18-4,35\end{array}$ & $\begin{array}{l}6 \\
5 \\
3\end{array}$ & $\begin{array}{ll}x & 2 \\
2 & x \\
2 & 2\end{array}$ & $\begin{array}{l}2 \\
x \\
2\end{array}$ & & & & & & $\begin{array}{l}8 \\
3\end{array}$ & & & $\begin{array}{ll}2 & 0 \\
5 & \mathrm{x} \\
2 & \mathrm{x}\end{array}$ & & & & $\begin{array}{l}0 \\
0 \\
0\end{array}$ & & 0 & \begin{tabular}{|l|l|} 
& \\
$x$ & 3 \\
0 & 3
\end{tabular} & $\begin{array}{ll}1 & 0 \\
3 & 0 \\
3 & 0\end{array}$ & $\begin{array}{ll}0 & 0 \\
0 & 0 \\
0 & 0\end{array}$ & $\left\{\begin{array}{l}x \\
0 \\
0\end{array}\right.$ & $\begin{array}{l}0 \\
x \\
0\end{array}$ & $\begin{array}{l}0 \\
0 \\
0\end{array}$ & $\begin{array}{ll}0 & 0 \\
0 & 0 \\
0 & 0\end{array}$ & & & & & $\begin{array}{l}0 \\
0 \\
1\end{array}$ & 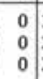 & & & \\
\hline
\end{tabular}


Table 1. (Continued).

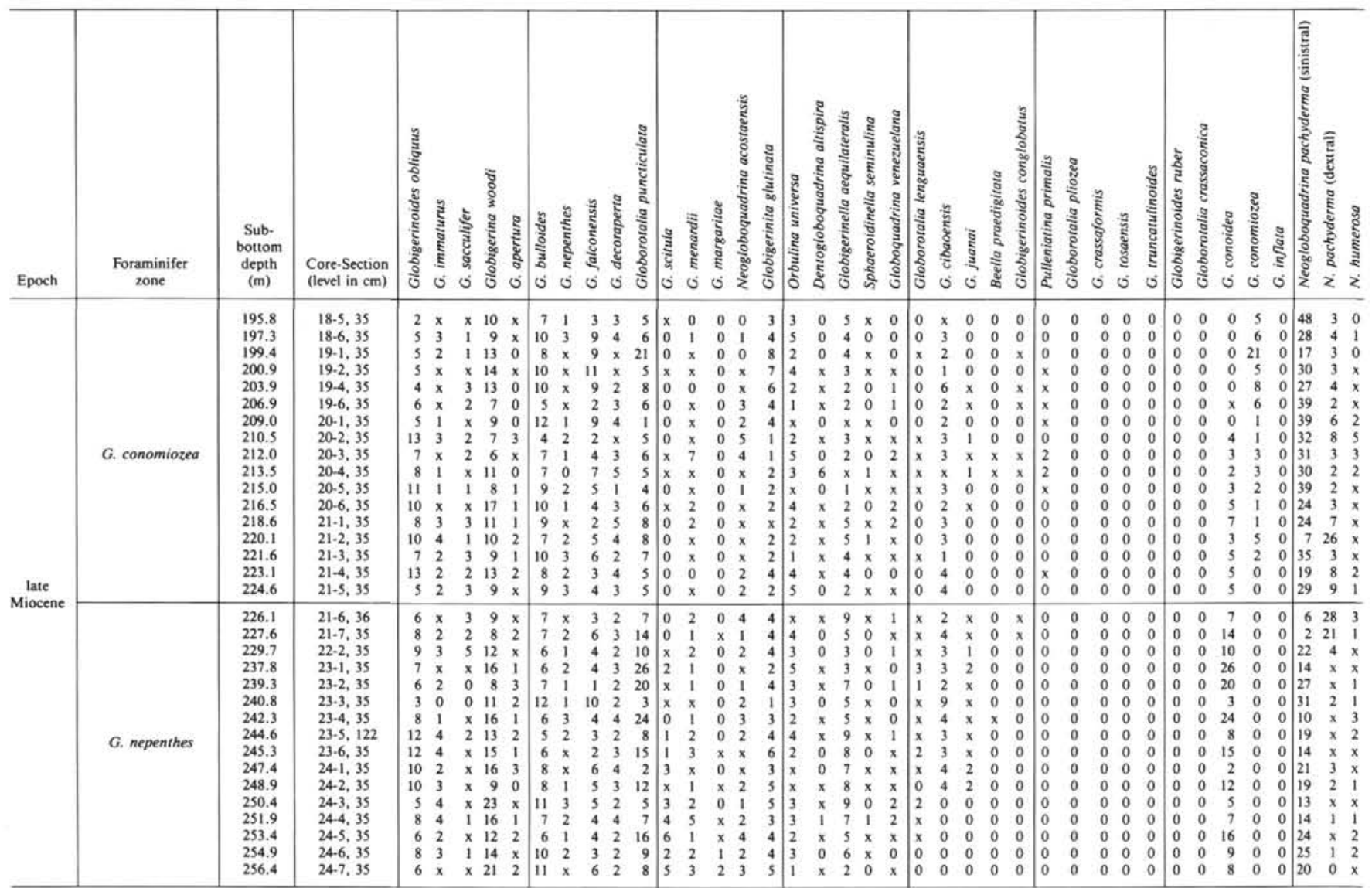

Note: $x$ refers to values of less than $1 \%$.

\section{Benthic Carbon Isotopes}

The benthic carbon isotope data for Cibicidoides kullenbergi are plotted against depth in Figure 10 and against time in Figure 11. We will continue to discuss the data within a chronological framework. The benthic carbon isotope record, except in the lower part of the sequence, is very similar throughout the late Neogene, averaging between $\sim 0.7$ and $0.9 \%$, and exhibits moderately high variability. The most distinct change in the benthic carbon isotope record occurs in the late Miocene between 6.7 and 6.2 Ma, representing a dramatic shift in carbon isotope values from 1.35 to $0.45 \%$ (peak). Lighter carbon isotope values mark the remainder of the late Neogene sequence, although there is a temporary return to heavier average values from 5.2 Ma, until about 4.5 Ma.

\section{Planktonic Carbon Isotopes}

The general trends in the planktonic and benthic foraminiferal carbon isotope oscillations are quite similar during the late Miocene and the late Pliocene. However, during the early Pliocene there is a noticeable divergence between the planktonic and benthic records (Fig. 11). The individual oscillations tend to covary quite well throughout the entire record, although the magnitude is often different. The magnitude of the isotopic change in the planktonic record is usually greater than that shown in the benthic record. The planktonic carbon isotope record also exhibits a very large shift from 2.7 to $1.23 \%$, coincident with the benthic shift (6.7 to 6.2 Ma). Average $\delta^{13} \mathrm{C}$ values of about $1.73 \%$ are exhibited between 6.3 and $4.6 \mathrm{Ma}$, after which there is a shift to slightly lighter average values of about $1.6 \%$ through the remainder of the Pliocene. Superimposed upon this lightening is a conspicuous lightening in $\delta^{13} \mathrm{C}$ of $1.0 \%$ in the early Pliocene at $4.2 \mathrm{Ma}$ (Fig. 11). Carbon isotope analyses were performed on Globorotalia puncticulata over the inter$\mathrm{val}$ of the planktonic foraminiferal carbon isotope spike at $\sim 4.2 \mathrm{Ma}$. The carbon isotope values of G. puncticulata are lighter than those of Globigerinoides sacculifer, although they display a similar depletion (Fig. 12). A second spike in planktonic $\delta^{13} \mathrm{C}$ occurs in the late Pliocene at 2.3 Ma.

\section{DISCUSSION}

\section{Paleoclimatic Analyses}

The location of Site 590 beneath the Subtropical Divergence, which separates warm-subtropical from temperate waters and is associated with divergence and upwelling, undoubtedly amplifies variability within the faunal assemblages recorded in the sediments. The fau- 


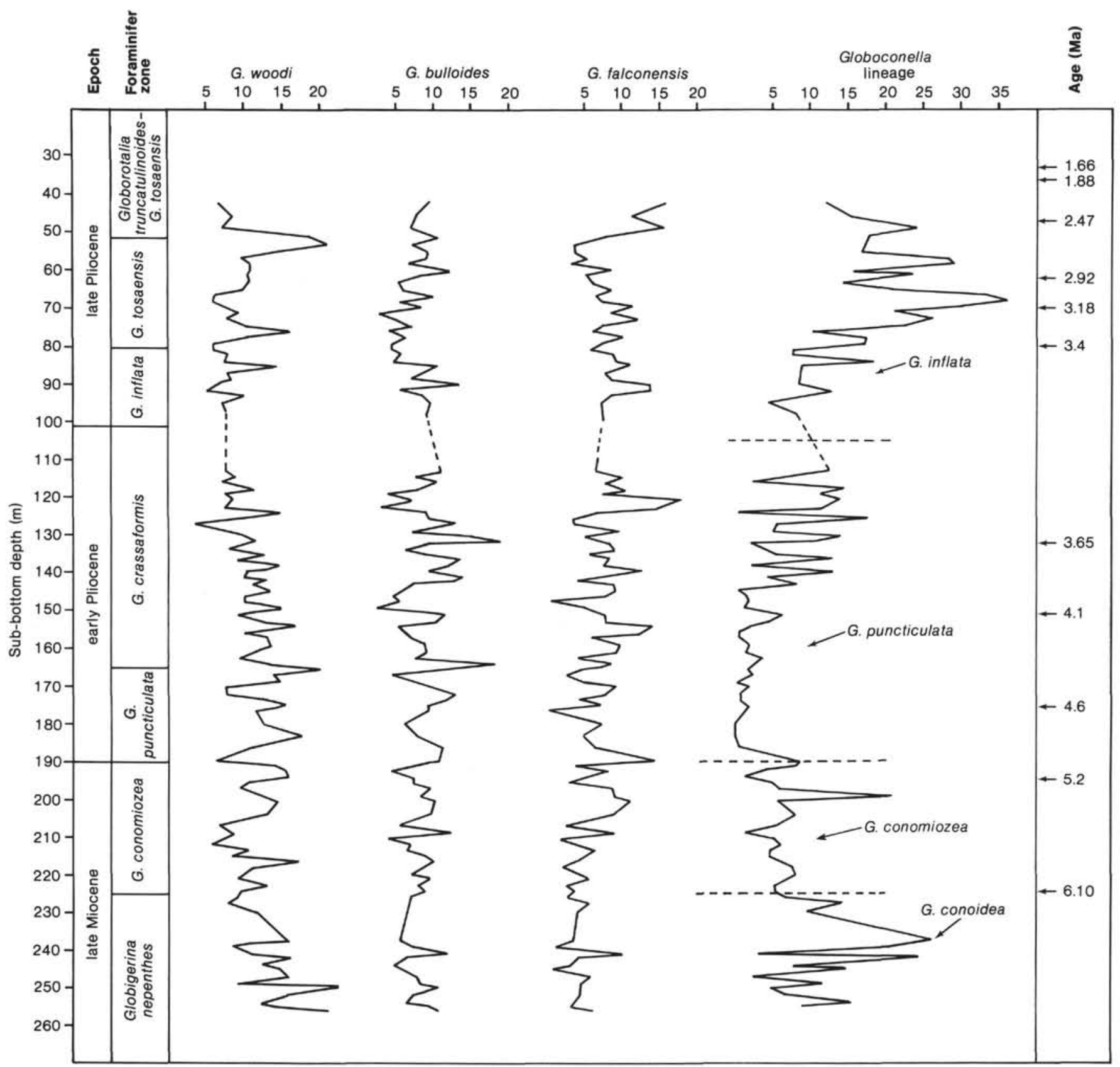

Figure 7. Frequency data for Globigerina woodi, G. bulloides, G. falconensis, and the Globoconella lineage (all in \%) plotted against depth at Site 590 .

nal assemblage at Site 590 is composed of both temperate and warm-subtropical forms; however, the temperate species are most abundant. Frequency oscillations of the cold-water form Neogloboquadrina pachyderma and the warm-subtropical fauna serve as sensitive temperature monitors in the late Miocene and early Pliocene and are used to evaluate changes in the temperature of surface waters during this time. The climatic interpretation is much more complex in the middle and late Pliocene at this site, since, as discussed later, the faunal data are reflecting local variability in surface-water temperature arising from changing intensities of upwelling that are not necessarily tied to global temperature changes.
The faunal data indicate that surface waters were relatively warm but cooling during the late Miocene between 6.3 and $5.6 \mathrm{Ma}$, as indicated by higher frequencies of the warm-subtropical fauna and increasing frequencies of $N$. pachyderma. Surface waters were generally much cooler in the latest Miocene and early Pliocene, between 5.6 and $4.3 \mathrm{Ma}$, when lowest frequencies of the warm-subtropical fauna coincide with highest frequencies of $N$. pachyderma. From 4.3 to about $3.3 \mathrm{Ma}$, both the warm subtropical fauna and $N$. pachyderma are equally abundant and exhibit highly variable frequency oscillations (Fig. 6). Higher frequencies of the cool-water form $N$. pachyderma in association with warm-water forms may 


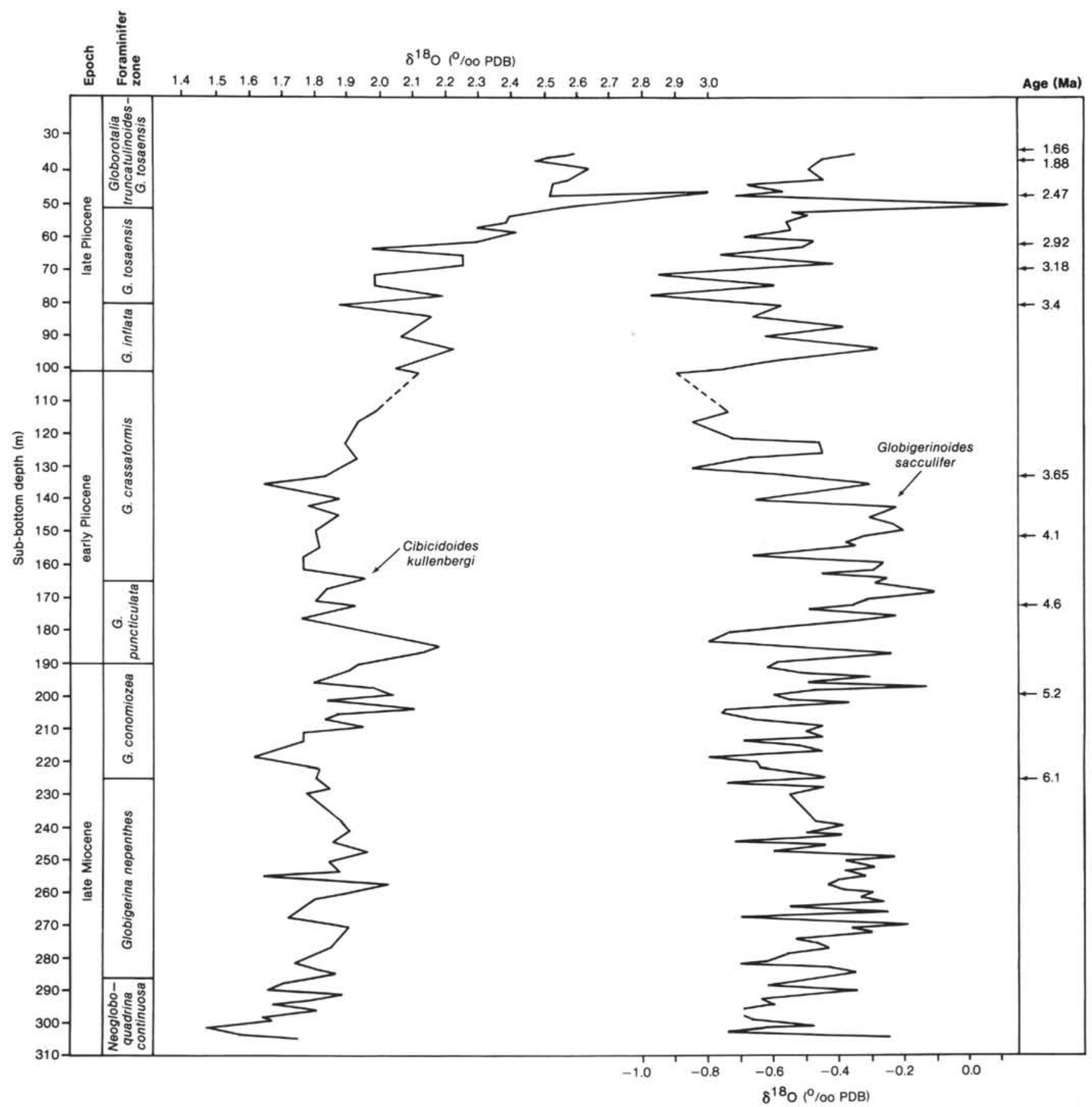

Figure 8. Planktonic and benthic foraminiferal $\delta^{18} \mathrm{O}$ plotted against depth at Site 590.

indicate variable temperatures, resulting from changing intensification of the Subtropical Divergence.

$N$. pachyderma and the warm-subtropical fauna both steadily decrease in frequency between 3.3 and $2.1 \mathrm{Ma}$, whereas Globorotalia inflata significantly increases (Fig. 7). There is no clear climatic interpretation in terms of temperature change represented in the faunal record at this time. The decrease in the frequency of the warmsubtropical forms and $N$. pachyderma may represent a decrease in the intensity of the Subtropical Divergence. Further evidence for this and for the resulting fluctuations within the faunal record will be presented later in a discussion of the increase in biogenic sedimentation rates in the early Pliocene.

Coiling direction changes of $N$. pachyderma at Site 590 exhibit no clear paleotemperature trends. A distinct switch in coiling direction occurs at about $4.3 \mathrm{Ma}$, in the early Pliocene, when the mode of coiling rapidly changes from dominantly sinistral to dominantly dextral. Kennett and Vella (1975) documented a similar, although more prolonged, switch in the coiling direction of N. pachyderma in the later part of the early Pliocene at Site 284 (Fig. 13), which is located in temperate waters south of Site 590. They suggested that this switch 


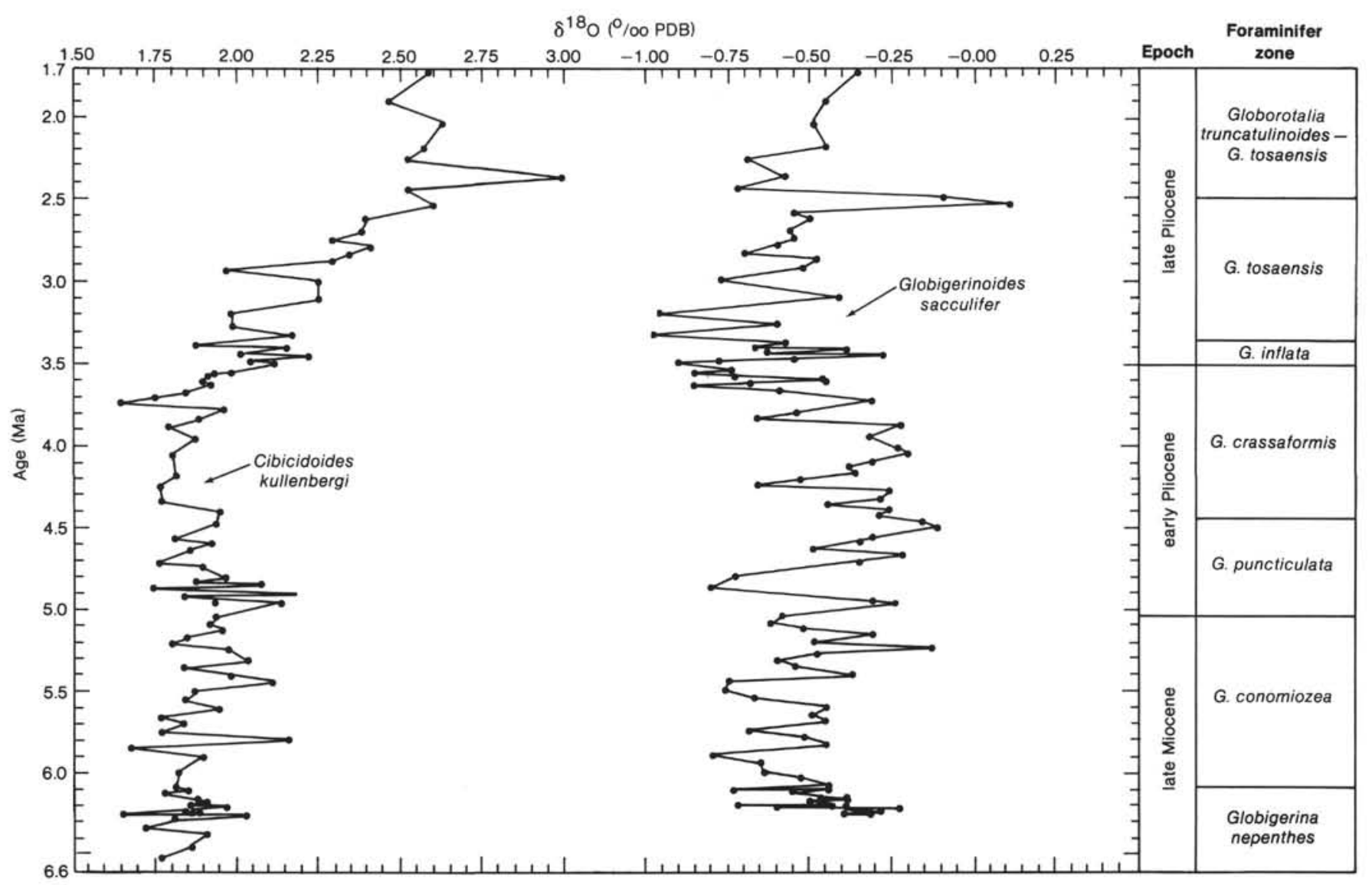

Figure 9. Planktonic and benthic foraminiferal $\delta^{18} \mathrm{O}$ plotted against age at Site 590.

reflected the southward migration of the dextral coiling populations as they increased their tolerance for cooler waters and displaced the sinistral populations to the south. Our data from Site 590 supports this interpretation, because the switch to dominantly dextral coiling occurs earlier in the northern site (Site 590) than in the more southern site (Site 284).

Planktonic foraminiferal oxygen isotope analyses were performed on Globigerinoides sacculifer, a species which is known to inhabit the upper $50 \mathrm{~m}$ of the water column and which contains symbiotic zooxanthellae (Bé et al., 1977). The planktonic foraminiferal oxygen isotope record only generally resembles the faunal climatic record, although both exhibit very high variability in the middle and late Pliocene. The oxygen isotope record exhibits no general trends through the late Neogene, with average values not varying by more than $0.3 \%$ between the late Miocene and the latest Pliocene (Fig. 9). High variability in the record most likely reflects temperature fluctuations associated with variable rates of upwelling at the Subtropical Divergence.

The climatic record at Site 590 is quite similar to that exhibited at Site 284 (Kennett and Vella, 1975) during the late Miocene through early Pliocene, with both sites indicating cool surface waters at that time. However, the records diverge in the middle Pliocene, when the faunal record at Site 590 seems to exhibit local variation while that of Site 284 indicates warmer conditions through the later part of the early Pliocene and cooler conditions during the late Pliocene in response to climatic cooling in the Northern Hemisphere (Kennett and Vella, 1975).

\section{Benthic Oxygen Isotope Trends}

The benthic foraminiferal oxygen isotope record at Site 590 reflects the late Neogene global climatic record, unlike the planktonic foraminiferal oxygen isotope record, which tends to monitor local variability in the temperature of surface waters. The late Neogene benthic foraminiferal oxygen isotope curve monitors oscillations in oceanic isotopic composition caused by continental ice growth and decay, as well as by changes in the temperature of intermediate water masses.

A $0.5 \%$ increase in benthic foraminiferal $\delta^{18} 0$ occurs between 5.6 and $4.8 \mathrm{Ma}$ in the latest Miocene and early Pliocene. A similar isotopic increase was documented at Site 284 (Shackleton and Kennett, 1975) (Fig. 14), and was interpreted to represent a $50 \%$ buildup of the West Antarctic ice sheet relative to the present day. This late Miocene isotopic enrichment has not always been found at other sites (Keigwin, 1979; Loutit, 1981), possibly because an unconformity was present at that time, or because sedimentation rates were low. However, further evidence for the late Miocene buildup of the West Antarctic ice sheet is based upon the northward expansion in the distribution of ice-rafted detritus in the Southern Ocean (Ciesielski et al., 1982); a late Miocene age of formation of the Ross Sea disconformity, which is believed to be due to the expansion of a grounded Ross ice shelf 
Table 2. Planktonic and benthic foraminiferal oxygen and carbon isotope data, Holes 590A, 590B.

\begin{tabular}{|c|c|c|c|c|c|}
\hline \multirow{2}{*}{$\begin{array}{c}\text { Core-Section } \\
\text { (interval in } \mathrm{cm} \text { ) }\end{array}$} & \multirow{2}{*}{$\begin{array}{l}\text { Depth } \\
\text { (m) }\end{array}$} & \multicolumn{2}{|c|}{$\begin{array}{c}\text { Globigerinoides } \\
\text { sacculifer }\end{array}$} & \multicolumn{2}{|c|}{$\begin{array}{c}\text { Cibicidoides } \\
\text { kullenbergi }\end{array}$} \\
\hline & & $\delta^{18} \mathrm{O}$ & ${ }_{\delta^{13} \mathrm{C}}$ & $\delta^{18} \mathrm{O}$ & ${ }_{\delta^{13} \mathrm{C}}$ \\
\hline \multicolumn{6}{|l|}{ Hole $590 \mathrm{~A}$} \\
\hline $1-6,35-36$ & 34.0 & -0.35 & 1.90 & 2.59 & 0.74 \\
\hline $2-1,35-36$ & 36.2 & -0.45 & 0.95 & 2.46 & 0.85 \\
\hline $2-3,35-36$ & 39.2 & -0.49 & 1.42 & 2.63 & 0.64 \\
\hline $2-5,35-36$ & 42.2 & -0.45 & 1.50 & 2.57 & 0.75 \\
\hline $2-6,35-36$ & 43.7 & -0.69 & 1.58 & 2.52 & 0.94 \\
\hline $3-1,35-36$ & 45.8 & -0.57 & 1.57 & 3.00 & 0.44 \\
\hline $3-2,35-36$ & 47.3 & -0.72 & 1.66 & 2.51 & 0.79 \\
\hline $3-3,35-36$ & 48.8 & -0.09 & 1.39 & & \\
\hline $3-4,35-36$ & 50.3 & 0.11 & 1.42 & 2.60 & 0.61 \\
\hline $3-5,35-36$ & 51.8 & -0.55 & 1.55 & & \\
\hline $3-6,35-36$ & 53.3 & -0.50 & 1.67 & 2.39 & 0.73 \\
\hline $4-1,35-36$ & 55.4 & -0.56 & 1.52 & 2.38 & 0.78 \\
\hline $4-2,35-35$ & 56.9 & -0.55 & 1.74 & 2.29 & 0.73 \\
\hline $4-3,35-36$ & 58.4 & -0.60 & 1.24 & 2.41 & 0.65 \\
\hline $4-4,35-36$ & 59.9 & -0.70 & 1.57 & 2.34 & 0.85 \\
\hline $4-5,35-36$ & 61.4 & -0.48 & 1.44 & 2.29 & 0.56 \\
\hline $4-6,35-36$ & 62.9 & -0.52 & 1.36 & 1.97 & 0.70 \\
\hline $5-1,110-112$ & 65.7 & -0.77 & 1.39 & 2.25 & 0.98 \\
\hline $5-3,110-112$ & 68.7 & -0.41 & 1.56 & 2.25 & 0.81 \\
\hline $5-5,110-112$ & 71.7 & -0.96 & 1.49 & 1.98 & 0.74 \\
\hline $6-1,55-57$ & 74.8 & -0.60 & 1.59 & 1.98 & 0.83 \\
\hline $6-3,55-57$ & 77.8 & -0.98 & 1.50 & 2.17 & 0.76 \\
\hline $6-5,55-57$ & 80.8 & -0.58 & 1.75 & 1.87 & 0.88 \\
\hline $7-1,35-36$ & 84.2 & -0.67 & 1.58 & 2.15 & 0.93 \\
\hline $7-3,35-36$ & 87.2 & -0.39 & 1.45 & & \\
\hline $7-5,35-36$ & 90.2 & -0.63 & 1.72 & 2.01 & 0.87 \\
\hline $8-1,103-104$ & 94.4 & -0.28 & 1.74 & 2.22 & 0.59 \\
\hline $8-3,35-36$ & 96.8 & -0.35 & 1.34 & & \\
\hline $8-5,35-36$ & 99.8 & -0.78 & 1.59 & 2.04 & 0.99 \\
\hline $8-6,35-36$ & 101.3 & -0.90 & 1.55 & 2.11 & 0.80 \\
\hline $10-1,35-36$ & 113.0 & -0.74 & 1.37 & 1.98 & 0.73 \\
\hline $10-3,35-36$ & 116.0 & -0.85 & 1.47 & 1.93 & 0.69 \\
\hline $10-5,35-36$ & 119.0 & -0.73 & 1.67 & 1.91 & 0.76 \\
\hline $11-1,35-36$ & 122.6 & -0.46 & 1.48 & 1.89 & 0.91 \\
\hline $11-3,35-36$ & 125.6 & -0.45 & 1.39 & & \\
\hline $11-4,35-36$ & 127.0 & -0.68 & 1.73 & 1.92 & 0.87 \\
\hline $11-6,35-36$ & 130.0 & -0.85 & 1.37 & & \\
\hline $12-1,35-36$ & 132.2 & -0.59 & 1.57 & 1.84 & 0.87 \\
\hline $12-3,35-36$ & 135.2 & -0.31 & 1.53 & 1.64 & 0.75 \\
\hline $12-5,35-36$ & 138.2 & -0.54 & 1.71 & & \\
\hline $12-6,35-36$ & 139.7 & -0.66 & 1.47 & 1.88 & 1.06 \\
\hline $13-1,35-36$ & 141.8 & -0.22 & 1.68 & 1.78 & 0.94 \\
\hline $13-3,35-36$ & 144.8 & -0.32 & 1.52 & 1.87 & 0.86 \\
\hline $13-5,35-36$ & 147.8 & -0.23 & 1.70 & & \\
\hline $13-6,35-36$ & 149.3 & -0.20 & 1.32 & & \\
\hline $14-1,35-36$ & 151.4 & -0.31 & 1.54 & 1.80 & 0.87 \\
\hline $14-2,35-36$ & 152.9 & -0.38 & 1.53 & & \\
\hline $14-3,35-36$ & 154.4 & -0.36 & 1.08 & 1.81 & 0.77 \\
\hline $14-4,35-36$ & 155.9 & -0.53 & 1.12 & & \\
\hline $14-5,35-36$ & 157.4 & -0.63 & 0.83 & 1.76 & 0.68 \\
\hline $14-6,35-36$ & 158.9 & -0.26 & 1.45 & & \\
\hline $15-1,35-36$ & 161.0 & -0.29 & 1.58 & 1.76 & 0.93 \\
\hline $15-2,35-36$ & 162.5 & -0.45 & 1.46 & & \\
\hline $15-3,35-36$ & 164.0 & -0.26 & 1.51 & 1.95 & 0.63 \\
\hline $15-4,35-36$ & 165.5 & -0.29 & 1.35 & & \\
\hline $15-5,35-36$ & 167.0 & -0.16 & 1.59 & 1.83 & 1.01 \\
\hline $15-6,35-36$ & 168.5 & -0.11 & 1.58 & & \\
\hline $16-1,35-36$ & 170.6 & -0.31 & 1.75 & 1.80 & 0.96 \\
\hline $16-2,35-36$ & 172.0 & -0.35 & 1.76 & 1.92 & 1.00 \\
\hline $16-3,35-36$ & 173.6 & -0.49 & 1.79 & 1.85 & 1.03 \\
\hline $16-4,35-36$ & 175.0 & -0.22 & 1.88 & & \\
\hline $16-5,35-36$ & 176.6 & -0.35 & 1.31 & 1.75 & 0.82 \\
\hline $17-1,35-36$ & 180.2 & -0.73 & 1.85 & 1.97 & 1.15 \\
\hline $17-3,35-36$ & 183.2 & -0.81 & 1.67 & & \\
\hline $17-3,121-122$ & 184.1 & & & 2.17 & 0.72 \\
\hline $17-5,35-36$ & 186.2 & -0.31 & 1.43 & 2.13 & 0.86 \\
\hline $17-5,121-122$ & 187.0 & -0.24 & 1.98 & & \\
\hline $18-1,35-36$ & 189.8 & -0.59 & 2.01 & 1.93 & 1.09 \\
\hline $18-2,35-36$ & 191.3 & -0.62 & 2.06 & 1.91 & 1.10 \\
\hline $18-3,35-36$ & 192.8 & -0.52 & 1.96 & & \\
\hline $18-4,35-36$ & 194.3 & -0.31 & 1.85 & 1.84 & 0.78 \\
\hline $18-5,35-36$ & 195.8 & -0.49 & 1.83 & 1.79 & 0.88 \\
\hline $18-6,35-36$ & 197.3 & -0.13 & 1.47 & 1.97 & 0.47 \\
\hline $18-6,121-122$ & 198.1 & -0.48 & 1.70 & & \\
\hline
\end{tabular}

Table 2. (Continued).

\begin{tabular}{|c|c|c|c|c|c|}
\hline \multirow{2}{*}{$\begin{array}{c}\text { Core-Section } \\
\text { (interval in cm) }\end{array}$} & \multirow{2}{*}{$\begin{array}{l}\text { Depth } \\
\text { (m) }\end{array}$} & \multicolumn{2}{|c|}{$\begin{array}{c}\text { Globigerinoides } \\
\text { sacculifer }\end{array}$} & \multicolumn{2}{|c|}{$\begin{array}{c}\text { Cibicidoides } \\
\text { kullenbergi }\end{array}$} \\
\hline & & $\delta^{18} \mathrm{O}$ & $\delta^{13} \mathrm{C}$ & $\delta^{18} \mathrm{O}$ & $\delta^{13} \mathrm{C}$ \\
\hline \multicolumn{6}{|l|}{ Hole 590A (Cont.) } \\
\hline $19-1,35-36$ & 199.4 & -0.60 & 1.63 & 2.03 & 0.68 \\
\hline $19-2,35-36$ & 200.9 & -0.50 & 1.93 & 1.83 & 0.84 \\
\hline $19-3,35-36$ & 202.4 & -0.37 & 1.65 & 1.97 & 0.69 \\
\hline $19-4,35-36$ & 203.9 & -0.75 & 1.54 & 2.10 & 0.59 \\
\hline $19-5,35-36$ & 205.4 & -0.76 & 1.78 & 1.86 & 0.88 \\
\hline $19-6,35-36$ & 206.9 & -0.37 & 2.01 & 1.83 & 0.70 \\
\hline $20-1,35-36$ & 209.0 & -0.45 & 1.28 & 1.94 & 0.51 \\
\hline $20-2,35-36$ & 210.5 & -0.50 & 1.59 & 1.76 & 0.66 \\
\hline $20-3,35-36$ & 212.0 & -0.45 & 1.76 & & \\
\hline $20-4,35-36$ & 213.5 & -0.69 & 2.11 & 1.76 & 0.88 \\
\hline $20-5,35-36$ & 215.0 & -0.52 & 1.68 & & \\
\hline $20-6,35-36$ & 216.5 & -0.45 & 1.59 & 1.67 & 0.65 \\
\hline $21-1,35-36$ & 218.6 & -0.80 & 1.84 & 1.61 & 0.80 \\
\hline $21-2,35-36$ & 220.1 & -0.65 & 1.57 & & \\
\hline $21-3,35-36$ & 221.6 & -0.64 & 1.59 & 1.81 & 0.50 \\
\hline $21-4,35-36$ & 223.1 & -0.53 & 1.90 & & \\
\hline $21-5,35-36$ & 224.6 & -0.44 & 1.97 & 1.80 & 0.93 \\
\hline $21-6,35-36$ & 226.1 & -0.74 & 2.11 & & \\
\hline $21-7,35-36$ & 227.6 & -0.45 & 1.76 & 1.84 & 0.71 \\
\hline $22-2,35-36$ & 229.7 & -0.55 & 2.01 & 1.77 & 0.67 \\
\hline $23-1,35-36$ & 237.8 & -0.47 & 1.73 & 1.87 & 0.69 \\
\hline $23-2,35-36$ & 239.3 & -0.39 & 1.78 & & \\
\hline $23-3,35-36$ & 240.8 & -0.50 & 1.68 & 1.90 & 0.75 \\
\hline $23-4,35-36$ & 242.3 & -0.39 & 1.54 & & \\
\hline $23-5,35-36$ & 244.3 & -0.72 & 1.57 & 1.85 & 0.70 \\
\hline $23-6,35-36$ & 245.3 & -0.17 & 1.65 & & \\
\hline $24-1,35-36$ & 247.4 & -0.60 & 1.26 & 1.96 & 0.48 \\
\hline $24-2,35-36$ & 248.9 & -0.23 & 1.56 & & \\
\hline $24-3,35-36$ & 250.4 & -0.38 & 1.24 & 1.84 & 0.45 \\
\hline $24-4,35-36$ & 251.9 & -0.29 & 1.58 & & \\
\hline $24-5,35-36$ & 253.4 & -0.38 & 1.62 & 1.87 & 0.46 \\
\hline $24-6,35-36$ & 254.9 & -0.32 & 1.85 & 1.54 & 0.60 \\
\hline $24-7,35-36$ & 256.4 & -0.40 & 1.56 & 1.85 & 0.54 \\
\hline $25-1,121-122$ & 257.0 & & & 2.02 & 0.69 \\
\hline $25-4,35-36$ & 261.5 & & & 1.80 & 0.77 \\
\hline $26-1,121-122$ & 267.4 & & & 1.71 & 0.79 \\
\hline $26-4,35-36$ & 270.1 & & & 1.90 & 0.70 \\
\hline $27-1,35-36$ & 276.2 & & & 1.85 & 0.82 \\
\hline $27-4,35-36$ & 280.7 & & & 1.76 & 1.02 \\
\hline
\end{tabular}

Hole 590B

\begin{tabular}{|c|c|c|c|c|c|}
\hline $28-5,35-36$ & 257.03 & -0.43 & 1.92 & & \\
\hline $28-6,35-36$ & 258.53 & -0.39 & 2.00 & & \\
\hline $29-1,35-36$ & 259.45 & -0.29 & 1.94 & & \\
\hline $29-2,35-36$ & 260.95 & -0.33 & 1.59 & & \\
\hline $29-3,35-36$ & 262.45 & -0.26 & 1.78 & & \\
\hline $29-4,35-36$ & 263.95 & -0.55 & 2.13 & & \\
\hline $29-5,35-36$ & 265.45 & -0.20 & 1.87 & & \\
\hline $29-6,35-36$ & 266.95 & -0.70 & 2.16 & & \\
\hline $30-1,35-36$ & 269.05 & -0.19 & 2.09 & & \\
\hline $30-2,35-36$ & 270.55 & -0.36 & 2.30 & & \\
\hline $30-3,35-36$ & 272.05 & -0.30 & 2.14 & & \\
\hline $30-4,35-36$ & 273.55 & -0.53 & 2.12 & & \\
\hline $30-5,35-36$ & 275.05 & -0.47 & 2.20 & & \\
\hline $30-6,35-36$ & 276.55 & -0.43 & 2.07 & & \\
\hline $31-1,35-36$ & 278.65 & -0.56 & 2.11 & & \\
\hline $31-2,35-36$ & 280.15 & -0.61 & 2.24 & & \\
\hline $31-3,35-36$ & 281.65 & -0.70 & 2.15 & 1.73 & \\
\hline $31-4,35-36$ & 283.15 & -0.43 & 2.11 & 1.78 & 0.93 \\
\hline $31-5,35-36$ & 284.65 & -0.35 & 2.15 & 1.86 & 1.10 \\
\hline $32-1,35-36$ & 288.45 & -0.62 & 2.18 & 1.69 & 1.06 \\
\hline $32-2,35-36$ & 289.75 & -0.34 & 2.23 & 1.65 & 1.12 \\
\hline $32-3,35-36$ & 291.25 & -0.49 & 2.13 & 1.88 & 0.93 \\
\hline $32-4,35-36$ & 292.75 & -0.64 & 2.27 & 1.77 & 0.98 \\
\hline $32-5,35-36$ & 294.25 & -0.60 & 2.49 & 1.66 & 1.02 \\
\hline $32-6,35-36$ & 295.75 & -0.69 & 2.35 & 1.81 & 0.97 \\
\hline $33-1,35-36$ & 297.85 & -0.69 & 2.48 & 1.63 & 1.26 \\
\hline $33-2,35-36$ & 299.35 & -0.67 & 2.14 & 1.66 & 1.19 \\
\hline $33-3,35-36$ & 300.85 & -0.48 & 2.54 & 1.49 & 1.28 \\
\hline $33-4,35-36$ & 301.35 & -0.62 & 2.67 & 1.46 & 1.37 \\
\hline $33-5,35-36$ & 302.85 & -0.74 & 2.57 & 1.55 & \\
\hline $33-6,35-36$ & 304.35 & -0.25 & 2.56 & 1.71 & 1.32 \\
\hline
\end{tabular}




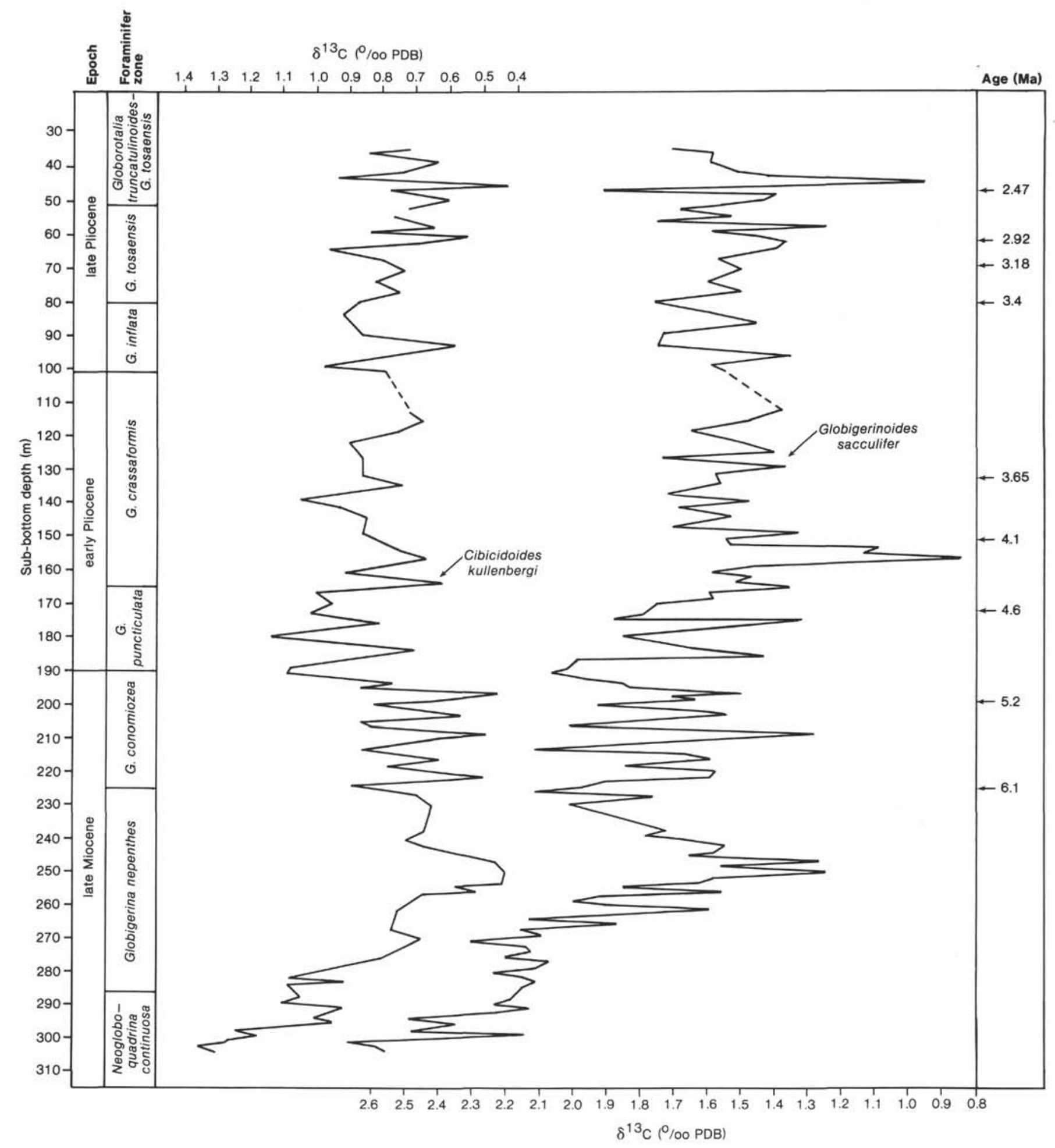

Figure 10. Planktonic and benthic foraminiferal $\delta^{13} \mathrm{C}$ plotted against depth at Site 590.

(Hayes and Frakes, 1975); the late Miocene initiation of glaciation in southern Argentina (Mercer and Sutter, 1982); and the northward expansion of the Polar Front and the siliceous biogenic belt (Tucholke et al., 1976; Brewster, 1980). The isotopic enrichment in the latest Miocene and early Pliocene at Site 590 must reflect both the buildup of ice on the West and East Antarctic ice sheets (Shackleton and Kennett, 1975; Ciesielski et al., 1982; Drewry, 1978) and a decrease in the temperature of the intermediate water mass as a result of climatic cooling at this time (Loutit, 1981).

The benthic foraminiferal oxygen isotope record indicates a return to lighter, more stable values by about 4.7 $\mathrm{Ma}$; these continue to about $3.7 \mathrm{Ma}$. This time of global 


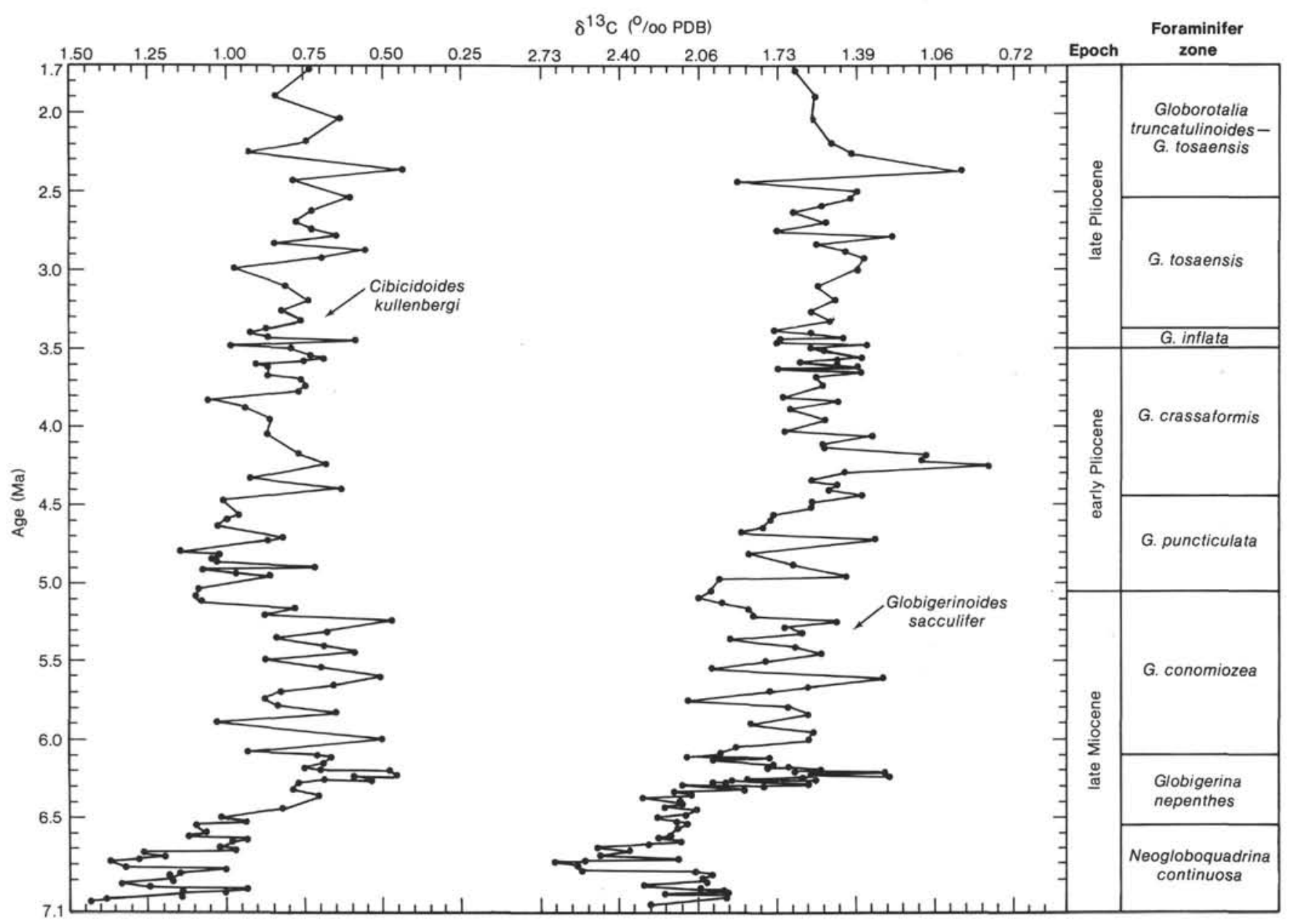

Figure 11. Planktonic and benthic foraminiferal $\delta^{13} \mathrm{C}$ plotted against age at Site 590.

climatic warmth and stability was also documented in the benthic foraminiferal oxygen isotope record from Site 284 (Shackleton and Kennett, 1975).

Two distinct changes occur in the benthic foraminiferal oxygen isotope record between 3.7 and $2.4 \mathrm{Ma}$. The first, between 3.7 and 3.4 represents a $0.3 \% 0$ average isotopic enrichment over a 0.3 m.y. period (Fig. 9). The second event, centered at about $2.7 \mathrm{Ma}$, represented a $0.5 \%$ average isotopic enrichment. Shackleton and Opdyke (1977) reported two oxygen isotope excursions in the late Pliocene from V28-179, the first at $3.2 \mathrm{Ma}$, the second at $2.5 \mathrm{Ma}$. The isotopic enrichment at $3.2 \mathrm{Ma}$ was originally considered to represent the initiation of Northern Hemisphere glaciation, whereas the second isotopic enrichment at $2.5 \mathrm{Ma}$ was believed to reflect an intensification of glaciation on the Northern Hemisphere (Shackleton and Opydke, 1977). Recent evidence suggests that the isotopic enrichment at 3.2 Ma may represent a brief excursion in ice volume rather than a permanent buildup, and/or a change in the character of bottom waters (Prell, 1982; Hodell et al., 1983). The second isotopic shift at $2.5 \mathrm{Ma}$ represents the true initiation of Northern Hemisphere glaciation, because the inception of ice-rafting at high latitudes in the North Atlantic has been redated at $2.5 \mathrm{Ma}$ by Backman (1979), and further isotopic and ice-rafting evidence from the North Atlan- tic suggest that large ice sheets did not accumulate on the Northern Hemisphere until about 2.5 Ma (Shackleton et al., 1984). The oxygen isotope enrichment centered at $3.6 \mathrm{Ma}$ at Site 590 may be correlated with the isotopic excursion noted by Shackleton and Opdyke (1977) at 3.2 Ma. The second isotopic enrichment at Site 590, centered at $2.7 \mathrm{Ma}$, reflects the initiation of Northern Hemisphere glaciation.

\section{Late Miocene Carbon Isotope Shift}

A permanent depletion in $\delta^{13} \mathrm{C}$ in the late Miocene is indicated in the planktonic and benthic foraminiferal carbon isotope records (Fig. 11). The carbon isotope shift in both planktonic and benthic foraminiferal $\delta^{13} \mathrm{C}$ is equal in magnitude $(0.5$ to $0.8 \%)$ and is considered isochronous with the globally recognized carbon isotope shift (Keigwin and Shackleton, 1980; Vincent et al., 1980; Haq et al., 1980) by the following criteria. The first appearance of the calcareous nannofossil Amaurolithus primus has been documented to occur prior to the globally recognized carbon shift at many sites (Haq et al., 1980), and at Site 590 this species first appears $15 \mathrm{~m}$ below the shift. Keigwin and Shackleton (1980) noted a distinct switch in the coiling direction of Neogloboquadrina acostaensis directly above the carbon shift in RC12-66, which is located in the equatorial Pacific. A switch in the coil- 


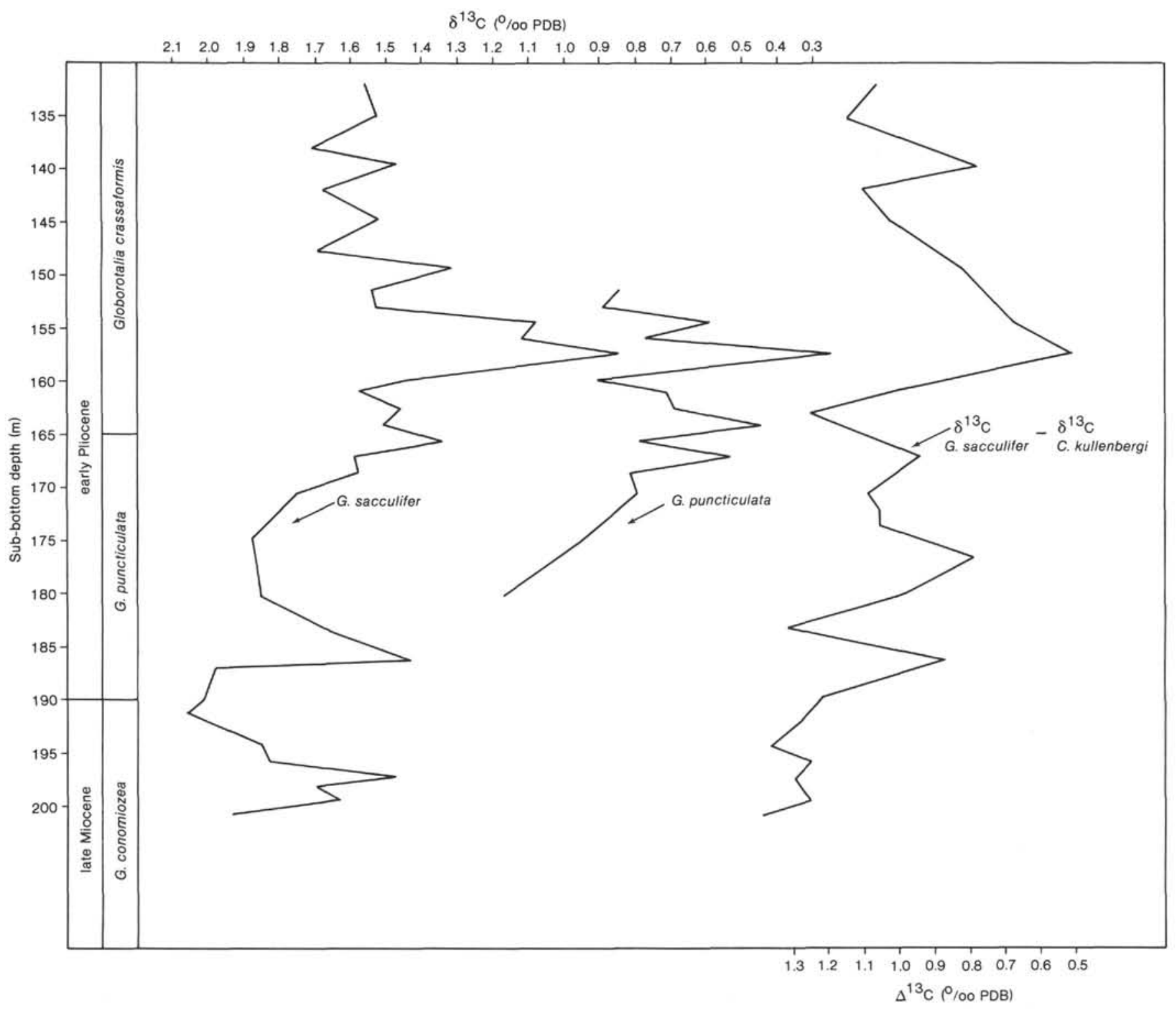

Figure 12. The $\delta^{13} \mathrm{C}$ of Globigerinoides sacculifer and Globorotalia puncticulata plotted against sub-bottom depth along with $\Delta^{13} \mathrm{C}\left(\delta^{13} \mathrm{C}\right.$ of $G$. sacculifer $-\delta^{13} \mathrm{C}$ of $\mathrm{G}$. puncticulata). The $\delta^{13} \mathrm{C}$ of $\mathrm{G}$. sacculifer is corrected to the $\delta^{13} \mathrm{C}$ of total $\mathrm{CO}_{2}$ of seawater using the correction factor of Williams et al., (1977). The $\delta^{13} \mathrm{C}$ of Cibicidoides kullenbergi is considered to be close to the $\delta^{13} \mathrm{C}$ of total $\mathrm{CO}_{2}$ of seawater (Belanger et al., 1981). $\Delta^{13} \mathrm{C}$ represents the gradient between surface and intermediate water $\delta^{13} \mathrm{C}$.

ing direction of $N$. pachyderma occurs directly above the carbon shift at Site 590 and may be correlated with the switch noted at RC12-66. Finally, Loutit and Kennett (1979) and Loutit (1981) noted that the first appearance of $G$. conomiozea occurred directly above the carbon shift at both the Blind River section in New Zealand and at DSDP Site 281. The first appearance of $G$. conomiozea is $40 \mathrm{~m}$ above the carbon shift at Site 590 . Loutit and Kennett (1979) assigned an age of 6.3 Ma to the late Miocene carbon isotope shift using a paleomagnetically dated marine section in New Zealand, whereas, Haq et al. (1980) assigned an age of 6.1 Ma, also using paleomagnetic stratigraphy.

\section{Early Pliocene Increased Biogenic Sedimentation}

A two-fold increase in biogenic sedimentation rates has been documented at Site 590 beginning at $\sim 4.2 \mathrm{Ma}$ and continuing to $\sim 3.2 \mathrm{Ma}$. This increase was also noted at other Leg 90 sites and is believed to reflect a true increase rather than a chronological problem (site chapter, this volume). The increase was specifically enhanced at the latitude of Site 590 because it is located near the Subtropical Convergence, a zone of upwelling (Kennett et al., 1983). Both the stable isotopic analyses and faunal data from Site 590 indicate that the character of surface waters changed at about the same time as the increase in biogenic sedimentation rates. The faunal data are dominantly composed of both warm- and cool-water taxa and the frequency oscillations are highly variable, a combination normally indicative of seasonal upwelling (Thiede, 1983). The planktonic foraminiferal oxygen isotope values are also highly variable, and heaviest average values are exhibited during this time, which may also indicate upwelling of cooler waters. The gradient be- 


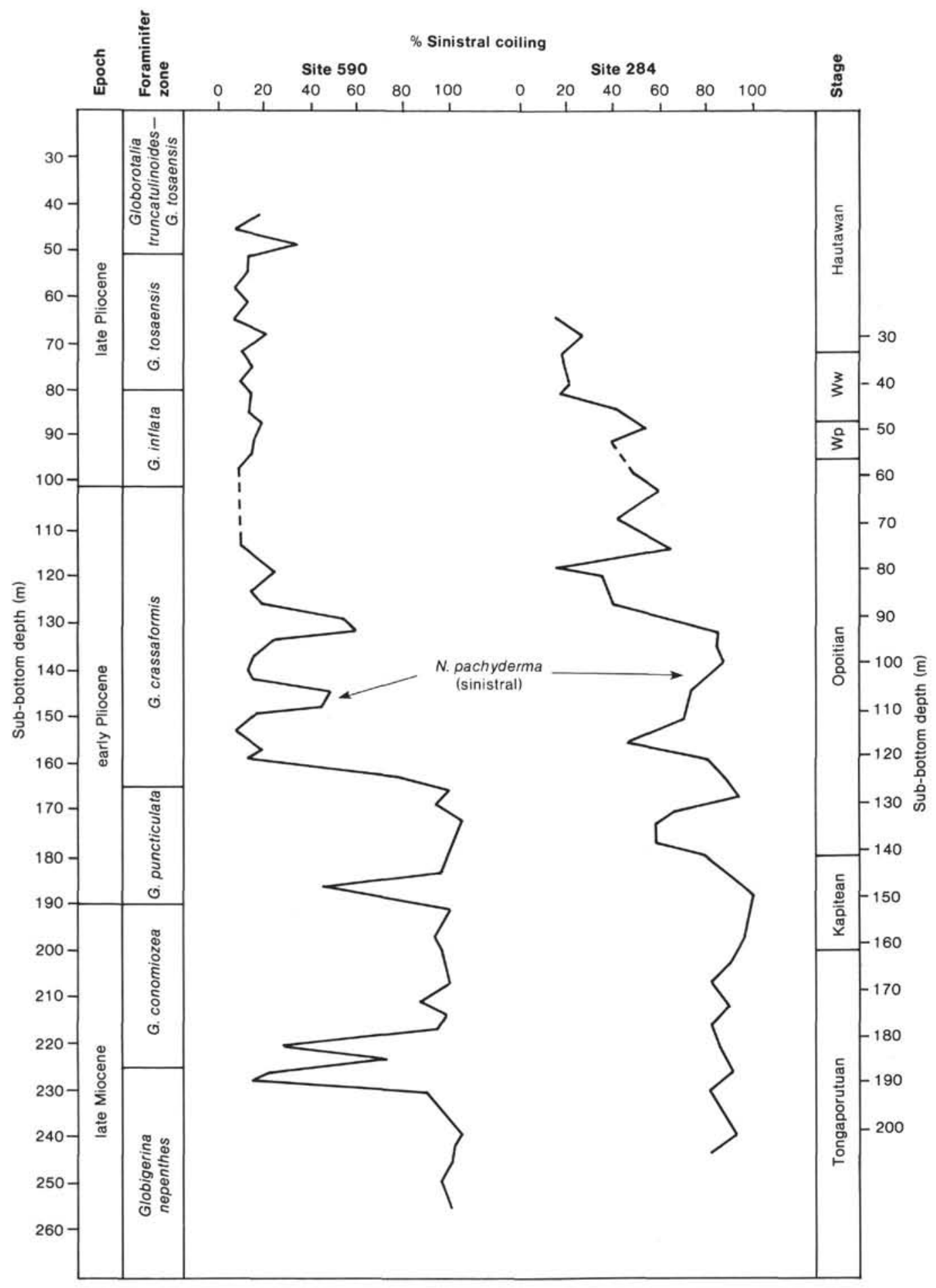

Figure 13. The coiling ratio of Neogloboquadrina pachyderma (\% sinistral) from Site 590, plotted against depth and the biostratigraphic zonation at Site 590, compared with the coiling ratio of $N$. pachyderma from Site 284, plotted against depth and the New Zealand stages (Kennett and Vella, 1975). The switch in coiling direction from dominantly sinistral to dominantly dextral occurs earlier at Site 590 than it does at Site 284. Ww = Mangapanian; $\mathrm{Wp}=$ Waipipian.

tween surface and intermediate-water $\delta^{18} \mathrm{O}\left(\Delta^{18} \mathrm{O}\right.$; Fig. 15) decreases during the interval of higher biogenic sedimentation rates. The $\delta^{13} \mathrm{C}$ gradient between surface and intermediate waters $\left(\Delta^{13} \mathrm{C}\right.$; Fig. 12$)$ also decreases at the same time as $\Delta^{18} \mathrm{O}$, suggesting that there was greater mixing between surface and intermediate waters at that time.
A marked depletion (spike) in planktonic foraminiferal $\delta^{13} \mathrm{C}(\sim 1.0 \%)$ at $4.2 \mathrm{Ma}$ heralds the beginning of lighter carbon isotope values through the Pliocene, and coincides with the onset of the increase in biogenic sedimentation rates. It appears that this spike is only a temporary event: extremely light $\delta^{13} \mathrm{C}$ values do not corre- 


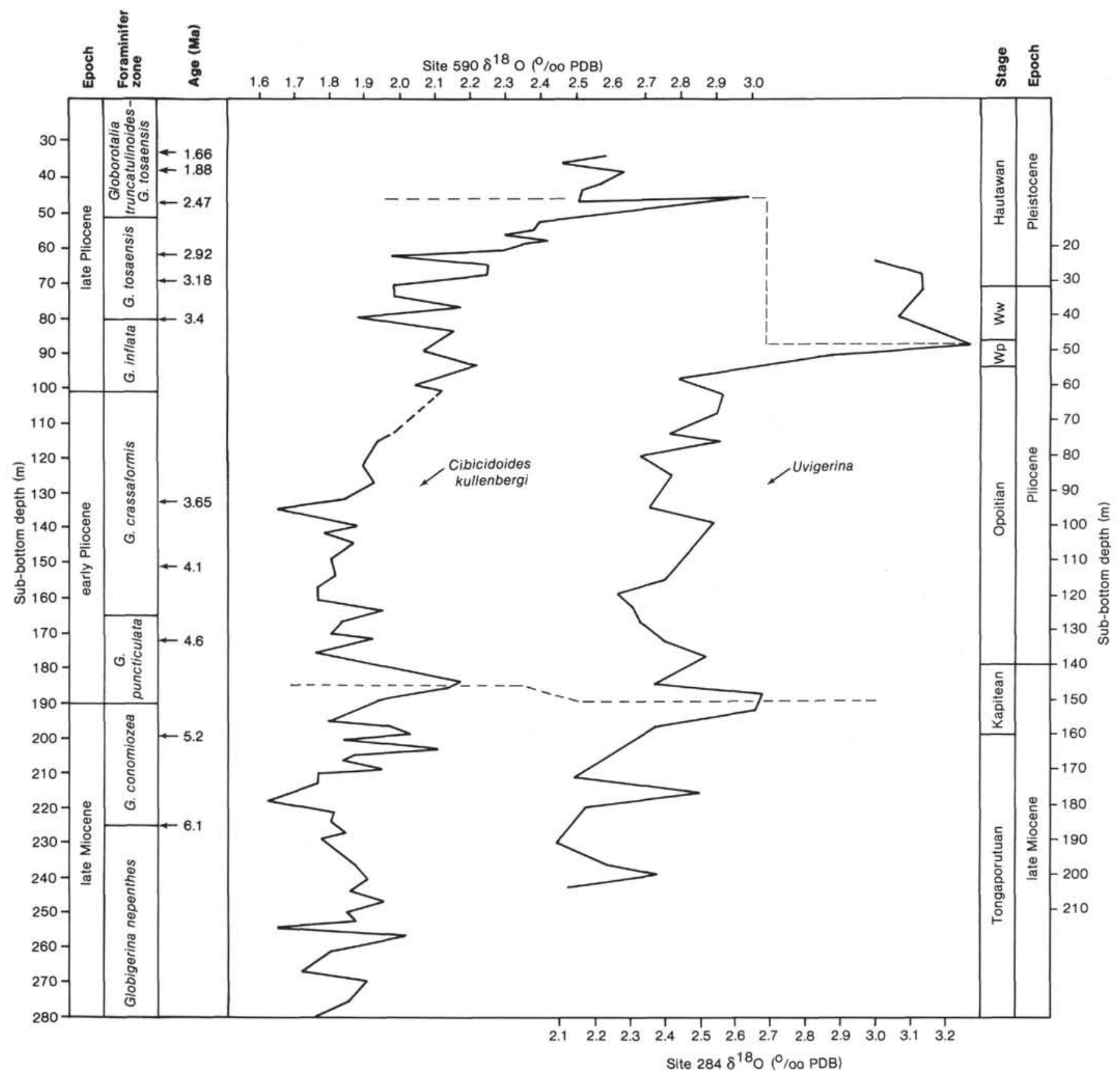

Figure 14. Benthic foraminiferal $\delta^{18} \mathrm{O}$ from Site 590 plotted against depth and the biostratigraphic zonation of Site 590, compared with benthic foraminiferal $\delta^{18} \mathrm{O}$ for Site 284 plotted against depth and the New Zealand stages (Kennett et al., 1979). Correlation lines refer to periods of maximum oxygen isotope enrichment. $W w=$ Mangapanian; $W p=$ Waipipian.

spond to the entire period of higher biogenic sedimentation rates. However, the average $\delta^{13} \mathrm{C}$ values are lighter through the remainder of the Pliocene. A major cause for change in the $\delta^{13} \mathrm{C}$ of total $\mathrm{CO}_{2}$ of surface waters is variation in surface-water productivity. The $\delta^{13} \mathrm{C}$ of total $\mathrm{CO}_{2}$ of surface water is a function of the photosynthetic respiration cycle (Kroopnick, 1974). Plants preferentially incorporate the lighter isotope ${ }^{12} \mathrm{C}$ during photosynthesis, leaving the total $\mathrm{CO}_{2}$ of surface waters enriched in the heavier isotope ${ }^{13} \mathrm{C}$. The lighter isotope ${ }^{12} \mathrm{C}$ is released to the water column during respiration, thereby producing light $\delta^{13} \mathrm{C}$ values in direct association with the $\mathrm{O}_{2}$ minimum zone (Kroopnick, 1974). During an upwelling event, the lighter isotope ${ }^{12} \mathrm{C}$ is brought back to the surface by the upward advection of water, thus lightening the $\delta^{13} \mathrm{C}$ of total $\mathrm{CO}_{2}$ of surface waters. Ganssen and Sarnthein (1983) investigated $\delta^{13} \mathrm{C}$ variations in planktonic foraminifers across the West African upwelling system. They found that Globorotalia inflata and Globigerinoides ruber both exhibited lighter $\delta^{13} \mathrm{C}$ values during periods of upwelling, reflecting the surfacing of waters enriched in ${ }^{12} \mathrm{C}$. Berger et al. (1978) found similar patterns in $\delta^{13} \mathrm{C}$ variations in G. ruber in the northwest African upwelling system, interpreting os- 


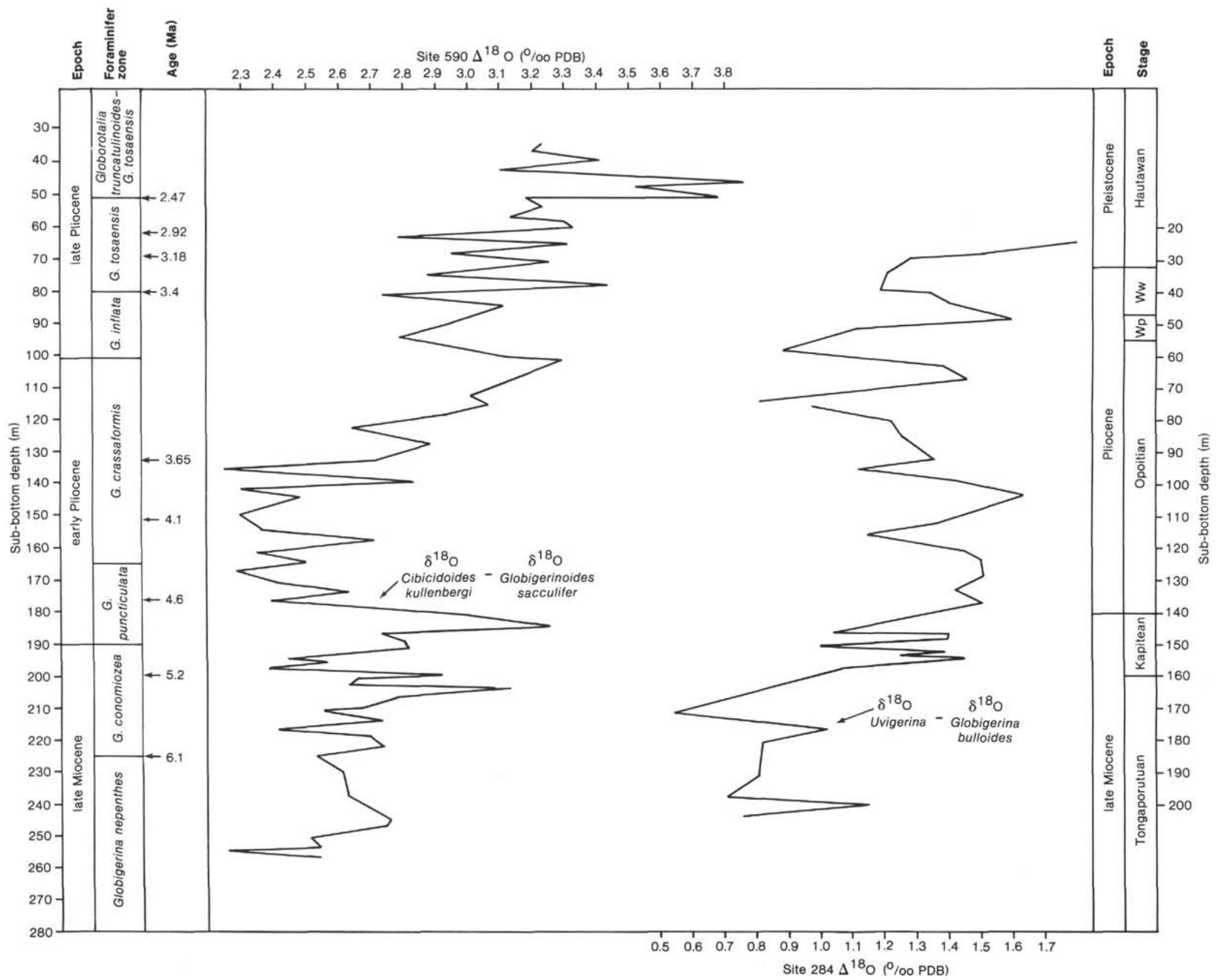

Figure 15. The gradient between surface and intermediate water $\delta^{18} \mathrm{O}\left(\Delta^{18} \mathrm{O}\right)$ for Site 590 plotted against sub-bottom depth and the biostratigraphic zonation of Site 590 , compared with the $\Delta^{18} \mathrm{O}$ for Site 284 plotted against depth and the New Zealand stages (Kennett et al., 1979). 
cillations in the $\delta^{13} \mathrm{C}$ of $G$. ruber to represent changes in upwelling intensity during the last glacial-interglacial cycle. On the other hand, Dunbar (1983) and Prell and Curry (1981) found that $\delta^{13} \mathrm{C}$ variations in Globigerina bulloides did not monitor upwelling events in the Santa Barbara Basin and the Arabian upwelling system, respectively. Prell and Curry (1981) suggested that large stratigraphic variations in planktonic foraminiferal $\delta^{13} \mathrm{C}$ may reflect changes in the global carbon cycle and not local surface-water productivity.

To test the validity of the spike in the $\delta^{13} \mathrm{C}$ of Globigerinoides sacculifer, a second species, Globorotalia puncticulata, was analyzed (Fig.12). G. puncticulata is a nonspinose species that is inferred to be a deeper dweller than Globigerinoides sacculifer (Bé, 1977). The plot shows that Globorotalia puncticulata also exhibits a marked decrease in $\delta^{13} \mathrm{C}$ of similar magnitude and coincident to the spike in the $\delta^{13} \mathrm{C}$ of Globigerinoides sacculifer. The marked depletion in $\delta^{13} \mathrm{C}$ at $\sim 4.2 \mathrm{Ma}$, recorded in the $\delta^{13} \mathrm{C}$ of two species of planktonic foraminifers, is interpreted as an indicator of upwelling in the early Pliocene at Site 590 . This interpretation is also supported by the planktonic foraminiferal oxygen isotope data and the faunal data, as stated earlier. The increase in biogenic sedimentation rates may therefore be associated with upwelling in the early Pliocene, which in turn increased the biogenic productivity of surface waters and produced a highly variable faunal record.

The increase in biogenic productivity is reflected mainly in the calcareous nannofossils, rather than the siliceous taxa, as normally occurs in areas of oceanic upwelling. This may be due to the nature of the upwelling regime. Most areas of siliceous biogenic productivity occur where cold, nutrient-rich, intermediate waters are upwelled. However, at the Subtropical Divergence, the upwelling is occurring over a shallow-water pedestal; thus the source of the upwelling waters is much shallower, and the water is warmer than at most oceanic upwelling regimes, such as the Polar Front, the Equatorial Divergence, and the Eastern Boundary Current systems. It remains to be seen what caused this increase in productivity during a time of known relative global climatic warmth and stability.

\section{CONCLUSIONS}

1. Site 590 is located under the present-day position of the Subtropical Divergence, which separates temperate surface water masses to the south from warm-subtropical water masses to the north. We have studied the paleoceanographic evolution of this site from the late Miocene $(\sim 7.0 \mathrm{Ma})$ to the latest Pliocene $(\sim 1.8 \mathrm{Ma})$, using quantitative changes in planktonic foraminifers and planktonic and benthic foraminiferal stable isotopes. Excellent core recovery, a shallow depth of deposition, and excellent microfossil preservation make this site an exceptional location for studying late Neogene paleoceanographic evolution.

2. A permanent depletion in both benthic and planktonic foraminiferal $\delta^{13} \mathrm{C}$ occurs between 6.7 and $6.2 \mathrm{Ma}$, and can be correlated to the globally recognized carbon isotope shift by the criteria which have been associated with the shift in various other localities (Loutit, 1981; Keigwin and Shackleton, 1980; Haq et al., 1980). The shift is located $40 \mathrm{~m}$ below the first appearance of Globorotalia conomiozea at $6.1 \mathrm{Ma}$; it is directly below a conspicuous change in the coiling direction of Neogloboquadrina pachyderma at $6.0 \mathrm{Ma}$; it is $15 \mathrm{~m}$ above the first appearance of Amaurolithus primus at $6.5 \mathrm{Ma}$, and is of comparable magnitude ( 0.5 to $0.8 \%$ ) to the shift at other sites.

3. The paleoclimatic record from Site 590 indicates that surface waters were cool in the latest Miocene and early Pliocene between 5.6 and $4.3 \mathrm{Ma}$, as indicated by high frequencies of $N$. pachyderma and low frequency of the warm-subtropical fauna. However, the paleoclimatic interpretation is ambiguous for the remainder of the sequence, since the faunal and planktonic foraminiferal oxygen isotopic data both appear to be reflecting local surface-water temperature variability resulting from interaction with the Subtropical Divergence and not global paleotemperature change.

4. A $0.5 \% 0$ enrichment in benthic foraminiferal $\delta^{18} \mathrm{O}$ during the latest Miocene and early Pliocene, between 5.6 and $4.8 \mathrm{Ma}$, reflects the buildup of ice on the Antarctic continent and the cooling of Antarctic Intermediate Waters. The benthic foraminiferal $\delta^{18} \mathrm{O}$ record indicates a return to lighter, more stable values by about 4.6 $\mathrm{Ma}$; this continues to about $3.7 \mathrm{Ma}$.

5. An increase in biogenic sedimentation rates (37.7 to $83.3 \mathrm{~m} / \mathrm{m}$.y.) in the early Pliocene at $\sim 4.2 \mathrm{Ma}$ coincides with a decrease in the gradient between surface and intermediate-water $\delta^{13} \mathrm{C}$ and $\delta^{18} \mathrm{O}$, increased variability within the planktonic foraminiferal frequency data and oxygen isotope data, heaviest values in the planktonic foraminiferal oxygen isotope record, and a $1.0 \%$ lightening in the $\delta^{13} \mathrm{C}$ of two species of planktonic foraminifers. These events reflect localized upwelling at the Subtropical Divergence, which, in turn, produced an increase in the biogenic productivity of surface waters at Site 590 .

6. A two-step enrichment occurs in the benthic foraminiferal oxygen isotope record in the late Pliocene at Site 590. The first step represents an average enrichment of 0.3 (0.5 peak $) \%$, centered at $\sim 3.6 \mathrm{Ma}$. The second step represents a permanent enrichment of $0.5(0.8$ peak) $\%$ centered at $\sim 2.7 \mathrm{Ma}$. These two events may correspond to the well-documented two-step isotopic enrichment associated with late Pliocene climatic cooling and the initiation of Northern Hemisphere glaciation.

\section{ACKNOWLEDGMENTS}

The authors would like to thank R. C. Thunell and G. Keller for reviewing the manuscript and Karin Nelson and Robin Rice for general assistance. This research was supported by NSF grant OCE 8214937 (Marine Geology).

\section{REFERENCES}

Adams, C. G., Benson, R. H., Kidd, R. B., Ryan, W. B. F., and Wright, R. C., 1977. The Messinian salinity crisis and evidence of late Miocene eustatic changes in the world oceans. Nature, 269: 383-386.

Backman, J., 1979. Pliocene biostratigraphy of DSDP Sites 111 and 116 from the North Atlantic Ocean and the age of Northern Hemisphere glaciation. Stockholm Contrib. Geol, 32:115-137.

Bé, A. W. H., 1977. An ecological, zoogeographic, and taxonomic review of Recent planktonic foraminifera. In Ramsey A. T. S. (Eds.), 
Oceanic Micropaleontology (Vol. 1): New York (Academic Press), $1-88$.

Bé, A. W. H., Hemleben, C. Anderson, O. R., Spindler, M., Hacunda, J., and Tuntivate-Choy, S., 1977. Laboratory an field observations of living planktonic foraminifera. Micropaleonotology, 23: 155-179.

Belanger, P. E., Curry, W. B., and Matthews, R. K., 1981. Core-top evaluation of benthic foraminiferal isotope ratios for paleo-oceanographic interpretations. Palaeogeogr. Palaeoclimatol. Palaeoecol., 33:205-220.

Berger, W. H., Diester-Haass, L., and Killingley, J. S., 1978. Upwelling off Northwest Africa: the Holocene decrease as seen in carbon isotopes and sedimentological indicators. Oceanol. Acta, 1:1-7.

Berggren, W. A., Kent, D. V., and Van Couvering, J. A., in press. Neogene geochronology and chronostratigraphy. In Snelling, N. J., (Ed.), Geochronology and Geologic Time Scale. Geol. Soc. London, Spec. Paper.

Brewster, N. A., 1980. Cenozoic biogenic silica sedimentation in the Antarctic Ocean. Geol. Soc. Am. Bull., 91:337-347.

Ciesielski, P. F., Ledbetter, M. T., and Elwood, B. B., 1982. The development of Antarctic glaciation and the Neogene paleoenvironment of the Maurice Ewing Bank. Mar. Geol., 46:1-52.

Denham, R. N., and Crook, F. G., 1976. The Tasman Front. N. Z. J. Mar. Freshw. Res., 10:15-30.

Drewry, D. J., 1978. Aspects of the early evolution of West Antarctic ice. In Van Zinderen Balker, E. M., (Ed.), Antarctic Glacial History and World Paleoenvironments: Rotterdam (A. A. Balkema), pp. 25-32.

Dunbar, R. B., 1983. Stable isotope record of upwelling and climate from Santa Barbara Basin, California. In Suess, E., and Thiede, J. (Eds.), Costal Upwelling, Its Sediment Record: New York (Plenum Press), pp. 217-246.

Ganssen, G., and Sarnthein, M., 1983. Stable-isotope composition of foraminifers: The surface and bottom water record of costal upwelling. In Suess, E., and Thiede, J. (Eds.), Costal Upwelling, Its Sediment Record: New York (Plenum Press), pp. 99-124.

Haq, B. U., Worsley, T. R., Burckle, L. H., Douglas, R., Keigwin, L. D., Jr., Opdyke, N. D., Savin, S. M., Sommer, M. A., II, Vincent, E., and Woodruff, F., 1980. Late Miocene marine carbon isotopic shift and synchroneity of some phytoplanktonic biostratigraphic events. Geology, 8:427-431.

Hayes, D. E., and Frakes, L. A., 1975. General synthesis, DSDP Leg 28.In Hayes, D. E., Frakes L. A., et al., Init. Repts. DSDP, 28: Washington (U.S. Govt. Printing Office), 919-942.

Hodell, D. A., Kennett, J. P., and Leonard, K. A., 1983. Climatically induced changes in vertical water mass structure of the Vema Channel during the Pliocene; Evidence from DSDP Holes 516A, 517, and 518. In Barker, P. F., Carlson, R. L., Johnson, D. A., et al., Init. Repts. DSDP, 72: Washington (U.S. Govt. Printing Office), 907-919.

Keigwin, L. D., Jr., 1979. Late Cenozoic stable isotopic stratigraphy and paleoceanography of DSDP sites from the east equatorial and central Pacific Ocean. Earth Planet. Sci. Lett., 45:361-382.

Keigwin, L. D., Jr., and Shackleton, N. J., 1980. Uppermost Miocene carbon isotope stratigraphy of a piston core in the equatorial $\mathrm{Pa}$ cific. Nature, 284:613-614.

Kennett, J. P., 1967. Recognition and correlation of the Kapitean Stage (Upper Miocene, New Zealand). N. Z. J. Geol. Geophys., 10: 1051-1063.

1973. Middle and late Cenozoic planktonic foraminiferal biostratigraphy of the southwest Pacific, DSDP Leg 21. In Burns, R. E., Andrews, J. E., et al., Init. Repts. DSDP, 21: Washington (U.S. Govt. Printing Office), 575-639.

1978. The development of planktonic biogeography in the Southern Ocean during the Cenozoic. Mar. Micropaleontol., 3: 301-345,

Kennett, J. P., Shackleton, N. J., Margolis, S. V., Goodney, D. E., Dudley, W. C., and Kroopnick, P. M., 1979. Late Cenozoic oxygen and carbon isotopic history and volcanic ash stratigraphy; DSDP Site 284, South Pacific. Am. J. Sci., 279:52-69.

Kennett, J. P., and Srinivasan, M. S., 1983. Neogene Planktonic Foraminifera: A Phylogenetic Atlas: Stroudsburg, Pa. (Hutchinson Ross Pub. Co.).

Kennett, J. P., and Vella, P., 1975. Late Cenozoic planktonic foraminifers and paleoceanography at DSDP Site 284 in the cool sub- tropical South Pacific. In Kennett, J. P., Houtz, R. E., et al., Init. Repts. DSDP, 29: Washington (U.S. Govt. Printing Office), 769-799.

Kennett, J. P., von der Borch, C., Baker, P. A., Barton, C. E., Boersma, A., et al., 1983. The South Pacific Cenozoic. Nature, 303: 18-19.

Killingley, J. S., Johnson, R. F., and Berger, W. H., 1981. Oxygen and carbon isotopes of individual shells of planktonic foraminifera from Ontong-Java plateau, equatorial Pacific. Palaeogeogr. Palaeoclimatol. Palaeoecol., 33:193-204.

Kroopnick, P., 1974. The dissolved $\mathrm{O}_{2}-\mathrm{CO}_{2}-{ }^{13} \mathrm{C}$ system in the eastern equatorial Pacific. Deep-Sea Res. 21:211-227.

Loutit, T. S., 1981. Late Miocene paleoclimatology: Subantarctic water mass, Southwest Pacific. Mar. Micropaleontol., 6:1-27.

Loutit, T. S., and Kennett, J. P., 1979. Application of carbon isotope stratigraphy to late Miocene shallow marine sediments, New Zealand. Science, 204:1196-1199.

Mercer, J. H., and Sutter, J. F., 1982. Late Miocene-earliest Pliocene glaciation in southern Argentina: Implications for global ice-sheet history. Palaeogeogr. Palaeoclimatol. Palaeoecol., 38:185-206.

Prell, W. L., 1982. A reevaluation of the initiation of Northern Hemisphere glaciation at $3.2 \mathrm{~m}$.y. new isotopic evidence. Geol. Soc. Am. Abstr. Progr., 592 (Abstract).

Prell, W. L., and Curry, W. B., 1981. Faunal and isotopic indices of monsoonal upwelling: Western Arabian Sea. Oceanol. Acta, 4: 91-98.

Rio, D., Sprovier, R., Di Stefano, E., and Raffi, I., 1964. Globorotalia truncatulinoides (d'Orbigny) in the Mediterranean Upper Pliocene geologic record. Micropaleontology., 2:121-137.

Shackleton, N. J., Backman, J., Zimmerman, H., Kent, D. V., Hall, M. A., Roberts, D. G., Schnitger, D., Bauldauf, J. G., Desprairies, A., Homrighausen, R., Huddleston, P., Keene, J. B., Kaltenback, A. J., Krumsiek, K. A. O., Morton, A. C., Murray, J. W., and Westberg-Smith, J., 1984. Oxygen isotopic calibration of the onset of ice rafting and history of glaciation in the North Atlantic region. Nature, 307:620-623.

Shackleton, N. J., and Kennett, J. P., 1975. Late Cenozoic oxygen and carbon isotopic changes at DSDP Site 284: Implications for glacial history of the Northern Hemisphere and Antarctica. In Kennett, J. P., Houtz, R. E., et al., Init. Repts. DSDP, 29: Washington (U.S. Govt. Printing Office), 801-806.

Shackleton, N. J., and Opdyke, N. D., 1977. Oxygen isotopic evidence for early Northern Hemisphere glaciation. Nature, 270: 216-219.

Srinivasan, M. S., and Kennett, J. P., 1981. A Review of Neogene planktonic foraminiferal biostratigraphy: Applications in the equatorial and south Pacific. In Warme, J. E., Douglas, R. G., and Winterer, E. L. (Eds.), The Deep Sea Drilling Project: A Decade of Progress. Soc. Econ. Paleontol. Mineral. Spec. Publ., 32:395-432.

Stanton, B. R., 1979. The Tasman Front. N. Z. J. Mar. Freshw. Res., 138:201-214.

1981. An oceanographic survey of the Tasman Front. N. Z. J. Mar. Freshw. Res., 15:289-297.

Thiede, J., 1983. Skeletal Plankton and Nekton in upwelling water masses of northwestern South America and northwest Africa. In Suess, E., and Thiede, J. (Eds.), Coastal Upwelling, Its Sediment Record. New York (Plenum Press), pp. 183-207.

Tucholke, B., Hollister, C. D., Weaver, F. M., and Vennum, W. R., 1976. Continental Rise and abyssal plain sedimentation in the southeast Pacific Basin, Leg 35, DSDP. In Hollister, C. D., Craddock, C., et al., Init. Repts. DSDP, 35: Washington (U.S. Govt. Printing Office), 359-400.

Vail, P. R., Mitchum, R. M., Jr., and Thompson, S., III, 1977. Global cycles of relative changes of sea level. In Payton, C. E. (Eds.), Seismic Stratigraphy-Applications to Hydrocarbon Exploration, Am. Assoc. Petrol. Geol. Mem., 26:83-97.

Vincent, E., Killingley, J. S., and Berger, W. H., 1980. The magnetic Epoch-6 carbon shift: a change in the oceans ${ }^{13} \mathrm{C} /{ }^{12} \mathrm{C}$ ratio 6.2 million years ago. Mar. Micropaleontol., 5:185-203.

Williams, D. F., Sommer, M. A., and Bender, M. L., 1977. Carbon isotopic compositions of Recent planktonic foraminifera of the Indian Ocean. Earth Planet. Sci. Lett., 36:391-403.

Date of Initial Receipt: 9 August 1984

Date of Acceptance: 5 December 1984 